\title{
Approaches towards the synthesis of a novel class of 2-amino-5-arylazonicotinate, pyridazinone and pyrido[2,3-d]pyrimidine derivatives as potent antimicrobial agents
}

Hamada Mohamed Ibrahim ${ }^{1,2}$, Haider Behbehani ${ }^{{ }^{*}}$ and Mohamed H Elnagdi ${ }^{1}$

\begin{abstract}
Background: Despite significant progresses in antimicrobial therapy, infectious diseases caused by bacteria and fungi remain a major worldwide health problem because of the rapid development of resistance to existing antimicrobial drugs. Therefore, there is a constant need for new antimicrobial agents. There are a large number of heterocyclic derivatives containing nitrogen atoms that possess a broad spectrum of biological activities including pyridine and pyridazine, which are two of the most important heterocycles in medicinal chemistry.

Results: The reaction of 3-oxo-2-arylhydrazonopropanals 2 with ethyl cyanoacetate and malononitrile 3a,b has led to the formation of 2-amino-5-arylazo-6-aryl substituted nicotinates $8 \mathrm{a}-\mathrm{k}$ as sole isolable products when the aryl group in the arylazo moiety was substituted with an electron-withdrawing group like $\mathrm{Cl}, \mathrm{Br}, \mathrm{NO}_{2}$. The pyridazinones 10 were formed from the same reaction when the arylazo moiety was phenyl or phenyl substituted with an electron-donating group. The 2-aminoazonicotinates 8 were condensed with DMF-DMA to afford the amidines 13a, $\mathrm{b}$, which then were cyclized to afford the targeted pyrido[2,3-d]pyrimidine derivatives $15 \mathrm{a}, \mathrm{b}$, respectively. The structures of all new substances prepared in this investigation were determined by using X-ray crystallographic analysis and spectroscopic methods. Most of the synthesized compounds were tested and evaluated as antimicrobial agents and the results indicated that many of the obtained compounds exhibited high antimicrobial activity comparable to ampicillin, which was used as the reference compound.
\end{abstract}

Conclusion: A general rule for the synthesis of 2-amino-5-arylazo-6-aryl substituted nicotinic acid and pyridazinone was established using 3-oxo-2-arylhydrazonopropanal as a precursor. Moreover, a novel route to pyrido[2,3-d] pyrimidine was achieved. Most of the synthesized compounds were found to exhibit strong inhibitory effects on the growth of Gram-positive bacteria especially Bacillus subtilis. Compounds 1a, 8a-h, 10a-c, 15b and 16 showed a broad spectrum of antimicrobial activity against B. subtilis.

Keywords: Azonicotinates, Pyridazinones, Pyrido[2,3-d]pyrimidine, X-ray, Antimicrobial activity

\footnotetext{
* Correspondence: hidar@bahbahani.com

${ }^{1}$ Chemistry Department, Faculty of Science, Kuwait University, P.O. Box 5969,

Safat 13060, Kuwait

Full list of author information is available at the end of the article
} 


\section{Background}

The emergence and spread of antimicrobial resistance has become one of the most serious public health concerns across the world. Antimicrobial resistance refers to microorganisms that have developed the ability to inactivate, exclude, or block the inhibitory or lethal effects of antimicrobial agents [1]. Despite significant progress in antimicrobial therapy, infectious diseases caused by bacteria and fungi remain a major worldwide health problem because of the rapid development of resistance to the existing antimicrobial drugs (antibacterial and antifungal). In other words, the increasing use and misuse of existing antimicrobial drugs have resulted in the development of resistant pathogens. In particular, the emergence of multidrug-resistant Gram-positive and -negative bacteria has caused life-threatening infectious diseases in many countries. The chemical and biological study of heterocyclic compounds has been of interest for many years for medicinal and agricultural reasons. There are a large number of heterocyclic derivatives containing nitrogen atoms such as pyridine and pyridazine that possess a broad spectrum of biological activities including antimicrobial [2-6], anti-inflammatory and analgesic [7-9], anti-HIV [10], antiplasmodial [11], anti-tubercular $[3,12]$, antibacterial $[3,13]$, anticonvulsant [14,15], inhibition of cyclo-oxygenase [16], antidiabetic [17], antihypertensive [18], anticancer [19-22], inhibition of blood platelet aggregation [23], antidepressant and anxiolytic [24,25], antioxidant [26] and antifungal [27]. Thus, the extensive biological activities of pyridine and pyridazine make them important in the design of druglike molecules. Encouraged by the afore-mentioned findings and in a continuation of an ongoing program aimed at finding new structural leads with potential potent antibacterial and antifungal agents [28,29], this study describes the synthesis of a new class of 2-amino-5-arylazo-6-aryl substituted nicotinic acid, pyridazinone, and pyrido[2,3- $d]$ pyrimidine derivatives.

\section{Results and discussion}

\section{Synthetic chemistry}

The reaction of the 3-oxo-2-arylhydrazonopropanals 2 with the active methylene reagents has been investigated in the past [30]. Recently, it was shown that this reaction affords either arylazo-2-oxonicotinates 6 or pyridazinones 10 [31]. However, the factors that control the nature of the end product could not be defined. In the present article, we report the synthesis of several derivatives of $\mathbf{2}$ with electron-donating and -withdrawing substituents on the arylazo moiety and identified the exact structure of the products of their reaction with the active methylene reagents $\mathbf{3 a}, \mathbf{b}$. It could be concluded that the reaction of $\mathbf{3}$ with 2 having an electron-donating substituent on the arylazo moiety afforded only the pyridazinones $\mathbf{1 0}$ while reacting $\mathbf{3}$ with $\mathbf{2}$ having an electron-withdrawing substituent on the arylazo moiety either in the $p, m$, or $o$ position or a mix of them affords only the 2-amino-5-arylazo-6-aryl substituted nicotinic acid derivatives 8. Thus compounds 2a-k were prepared via coupling of $\mathbf{1}$ with aromatic diazonium salts [30] (cf. Scheme 1 and Figure 1). Reacting 2a-g with ethyl cyanoacetate $\mathbf{3 a}$ or with malononitrile $\mathbf{3 b}$ affords the 2-amino-5-arylazo-6-aryl substituted nicotinates $\mathbf{8 a - k}$ as confirmed from accurate mass determination and elemental analyses. Moreover, the structures were also confirmed from the X-ray single crystal structure determination for $\mathbf{8 a}, \mathbf{8 b}, \mathbf{8 c}$, and $\mathbf{8 h}$ (cf. Figures 2, 3, 4, and 5, Tables 1, 2, and Scheme 1). It is believed that initially the acyclic condensation products $\mathbf{4}$ were formed and then these cyclize to the pyranimine $\mathbf{5}$ that reacts with ammonia from the reaction medium to yield the acyclic intermediate 7 that further cyclizes into the final isolable 2 -aminonicotinic acid derivatives 8 . Under these conditions, no traces of the arylazo-2-oxonicotinates 6 or 2 hydroxy-5-arylazonicotinates were isolated as reported by Al-Mousawi et al. [31,32]. On the other hand, the reaction of $\mathbf{2 h}-\mathbf{k}$ having a phenyl or a phenyl substituted with an electron-donating group on the arylhydrazone moiety with 3a afforded the pyridazinones 10a-d. It is believed that also in this case, the acyclic intermediate 4 was formed and then cyclized via attack of the arylhydrazone moiety at $\mathrm{CN}$ to afford the pyridazine imine intermediate 9 that was hydrolyzed under the reaction conditions to yield the final isolable pyridazinone 10. The structure of $\mathbf{1 0}$ was also supported by both the classical analytical analyses and through the X-ray crystal structure determination for 10a (cf. Figure 6, Table 3, and Scheme 1). It is believed that the basicity of the hydrazone moiety of $\mathbf{2}$ controls the nature of the final product as it facilitates the reversible cyclization of the intermediate $\mathbf{4}$ and at the same time helps to stabilize the cyclized 9, thus allowing the hydrolysis step to proceed to form the pyridazinone 10. In contrast, cyclization of $\mathbf{4}$ is highly reversible and a competing cyclization reaction takes place resulting in formation of the pyranimine 5 , which in the presence of ammonium ion led to the formation of the stable aromatic 2-aminonicotinic acid derivatives $\mathbf{8}$.

The obtained arylazoaminonicotinates are interesting precursors for the synthesis of a variety of a novel arylazoheterocycles that may possess interesting biological activities. Reaction of the 2-amino-5-arylazonicotinates $\mathbf{8}$ with acetic anhydride afforded the mono- and the diacetylated products $\mathbf{1 1}$ and 12, respectively, depending upon the reaction time. The structures of the products 11a and 12 were confirmed by X-ray single crystal determination (cf. Scheme 2, Figures 7, 8).

Moreover, the 2-amino-5-arylazonicotinates 8 reacted with dimethylformamide dimethylacetal (DMF-DMA) to yield the corresponding amidines 13. The amidines 
13a,b reacted with ammonia in refluxing acetic acid to yield the corresponding pyrido[2,3- $d]$ pyrimidine derivatives $\mathbf{1 5 a}, \mathbf{b}$. The structures of these products were also confirmed by different spectroscopic analyses as illustrated in the experimental section. Furthermore, fusion of the azonicotinates $\mathbf{8 f}$ with thiourea afforded the corresponding pyrido[2,3- $d]$ pyrimidine derivatives 16 (cf. Scheme 3).

\section{Antimicrobial activity}

The novel chemical compounds synthesized in this study showed promising antimicrobial activities. In general, most of the tested compounds revealed better activity against Gram-positive rather than the Gram-negative bacteria and yeast. The results as depicted in Table 4 show strong activities against Gram-positive bacteria because all of the tested chemicals showed highly positive 


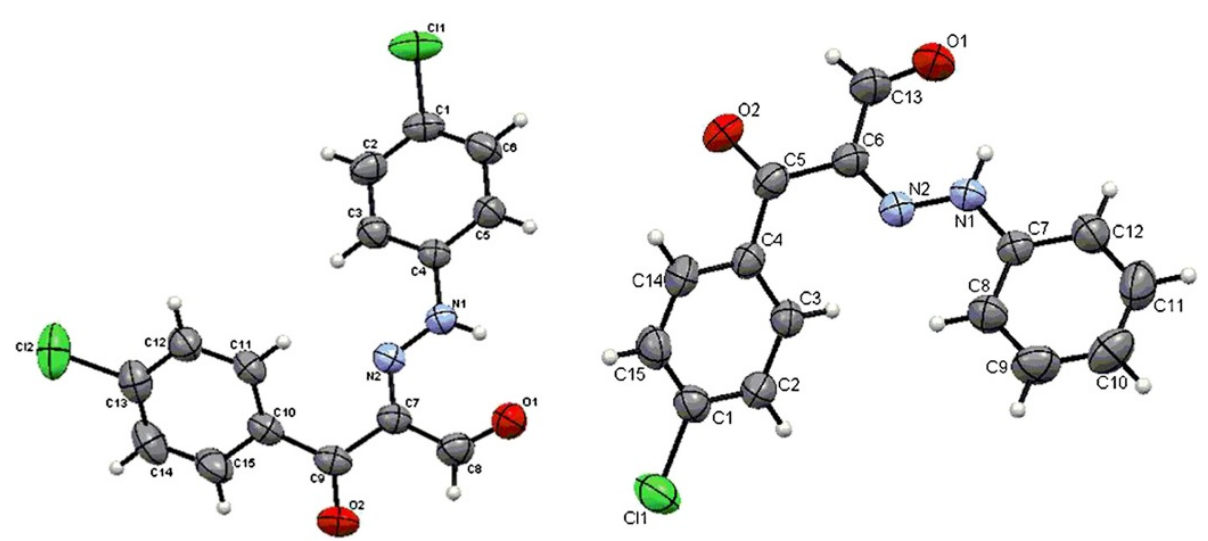

Figure 1 ORTEP plot of the X-ray crystallographic data determined for $2 \mathrm{a}$ and $2 \mathrm{~h}$.

antimicrobial activities against $B$. subtilis with inhibition zones $>10 \mathrm{~mm}$. Only the tested chemical 1a displayed strong inhibitory effects on the growth of Escherichia coli (Gram-negative bacteria), Bacillus subtilis, and Staphylococcus aureus (Gram-positive bacteria), which showed inhibition zones exceeding $10 \mathrm{~mm}$. It also strongly inhibited the growth of Candida albicans (yeast) while the cycloheximide did not inhibit growth of this yeast. None of the chemicals except 1a inhibited the growth of Gramnegative bacteria or yeast. Moreover compounds 2a, 2c, 2d, and $\mathbf{2 g}$ had high inhibitory activities against the Grampositive bacteria S. aureus. The tested chemicals 8a-h and 10a-c displayed very strong inhibitory effects toward the growth of the Gram-positive bacteria B. subtilis with inhibition zones exceeding the reference chemotherapeutic ampicillin (cf. Table 4). Compounds 8a and 10c were also nearly as active as ampicillin against $B$. subtilis ( $\mathrm{MIC}=$ $12.5 \mu \mathrm{g} / \mathrm{mL}$ ). It was found that transformation of the

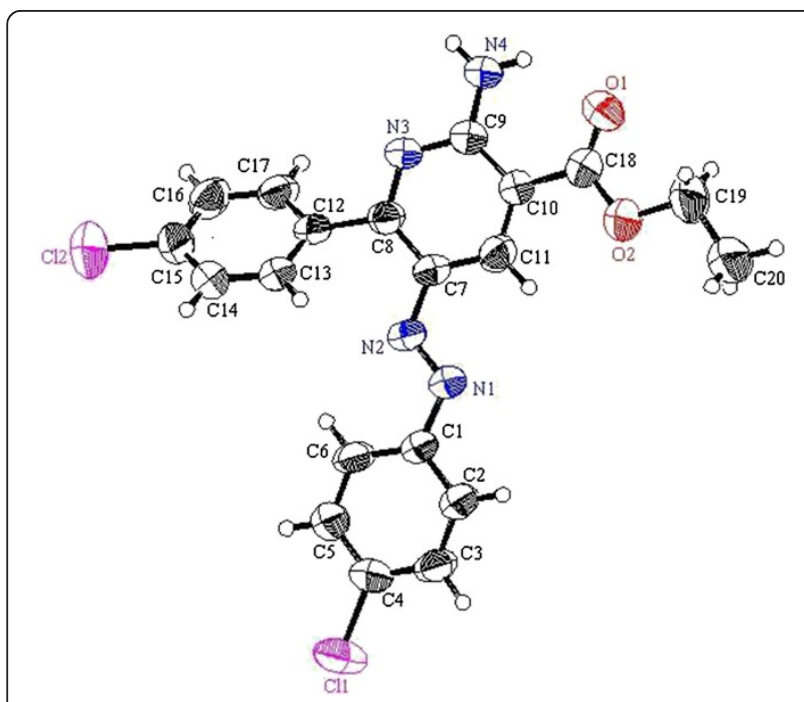

Figure 2 ORTEP plot of the X-ray crystallographic data determined for 8 . enaminones 1 into the corresponding arylhydrazonals 2 generally decreased the inhibitory effects while transformation of the latter into the corresponding 2amino-5-arylazo-6-aryl substituted nicotinates $\mathbf{8}$ or the pyridazinone $\mathbf{1 0}$ resulted in inhibition of the growth of only B. subtilis (Gram-positive bacteria) as revealed by the diameters of their inhibition zones. Conversely, conversion of the 2-aminoazonicotinates derivatives into the corresponding acetyl, diacetyl, or amidine derivatives exemplified by compounds 11, 12, and 13 unfortunately resulted in a decrease in the inhibitory effects but still had inhibition zones $>10 \mathrm{~mm}$. Fusing the pyridine ring into the bicyclic pyrido[2,3-d] pyrimidine derivatives $\mathbf{1 5 a}, \mathbf{b}$ and $\mathbf{1 6}$ enhanced the antimicrobial activity because the majority of these compounds were active against only the Grampositive bacteria $B$. subtilis and $S$. aureus.

- Structure activity relationship By comparing the experimental biological activity of the compounds reported in this study with their structures, the following structural activity relationship assumptions are postulated.

$>$ The pyridine or pyridazine moieties are necessary to observe the higher antibacterial activities towards the Gram-positive bacteria B. subtilis.

$>$ It is interesting to point out that for the azonicotinates $\mathbf{8}$ having an electron-withdrawing group in the arylazo moiety in the para-, metaand ortho- positions like compounds 8a-e or having two electron-withdrawing groups in the arylazo moiety as in $\mathbf{8 f}$ and $\mathbf{8 h}$ results in higher antibacterial activity as evidenced by the inhibition zones that were similar (Table 4), and from the minimum inhibitory concentration (MIC) values presented in Table 5. This indicates that high antimicrobial activity may be correlated with the low electron density of the ring systems and the role of an electron-withdrawing group in increasing the 


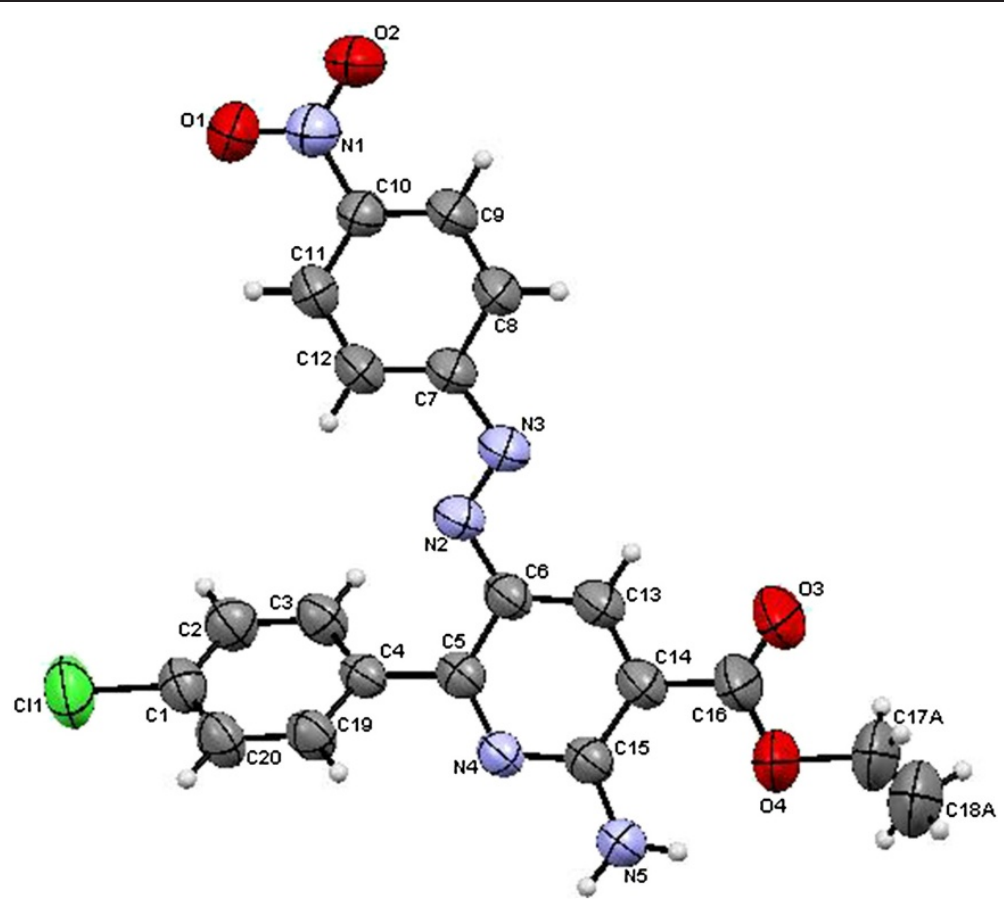

Figure 3 ORTEP plot of the X-ray crystallographic data determined for $8 \mathrm{~b}$.

antimicrobial potency is similar to the results of Sharma et al. [33].

$>$ It is worth mentioning that changing the COOEt group to a $\mathrm{CN}$ group as in $\mathbf{8} \mathbf{f}$ and $\mathbf{8 h}$ has no significant effect on the biological activity.

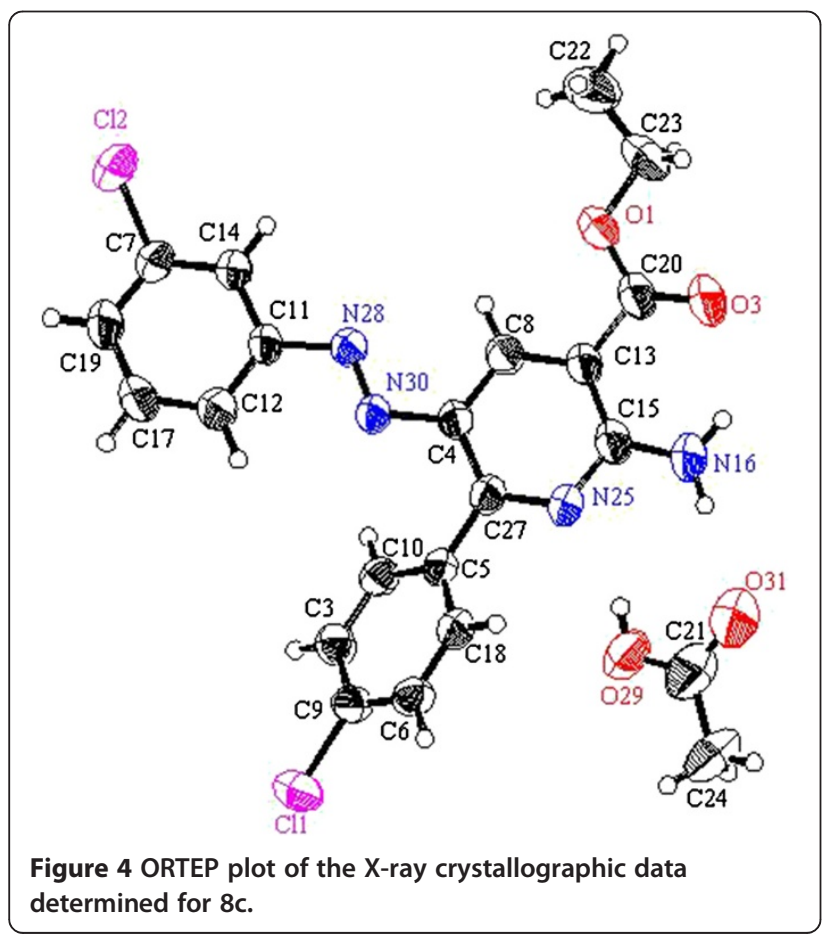

$>$ The presence of a Me or OMe (electrondonating group) in the aryl moiety in position 2 as in the pyridazine $\mathbf{1 0 b}, \mathbf{c}$ enhances the biological activity. $>$ Transformation of the azonicotinates $\mathbf{8}$ to the pyrido[2,3-d]pyrimidine derivatives $\mathbf{1 5 a}, \mathbf{b}$ and $\mathbf{1 6}$ does not significantly affect the biological activity against the Gram-positive bacteria B. subtilis.

\section{Experimental}

General

Melting points were recorded on a Griffin melting point apparatus and are reported uncorrected. IR spectra were recorded using $\mathrm{KBr}$ disks using a Perkin-Elmer System 2000 FT-IR spectrophotometer. ${ }^{1} \mathrm{H}-\mathrm{NMR}(400 \mathrm{MHz})$ or $(600 \mathrm{MHz})$ and ${ }^{13} \mathrm{C}-\mathrm{NMR}(100 \mathrm{MHz})$ or $(150 \mathrm{MHz})$ spectra were recorded at $25^{\circ} \mathrm{C}$ in $\mathrm{CDCl}_{3}$ or DMSO- $d_{6}$ as solvent with TMS as internal standard on a Bruker DPX 400 or 600 super-conducting NMR spectrometer. Chemical shifts are reported in ppm. Mass spectra were measured using a high resolution GC-MS (DFS) thermo spectrometers with EI (70 EV). Microanalyses were performed on a LECO CHNS-932 Elemental Analyzer. Follow up of the reactions and checking homogeneity of the prepared compounds was made by thin layer chromatography (TLC). All single crystal data collections were made either on Rigaku R-AXIS RAPID diffractometer

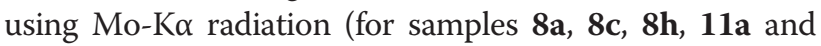
12) or on Bruker $\mathrm{X} 8$ Prospector using $\mathrm{Cu}-\mathrm{K} \alpha$ radiation (for compounds $\mathbf{2 a}, \mathbf{2 h}, \mathbf{8 b}$, and $\mathbf{1 0 a}$ ). The data were 


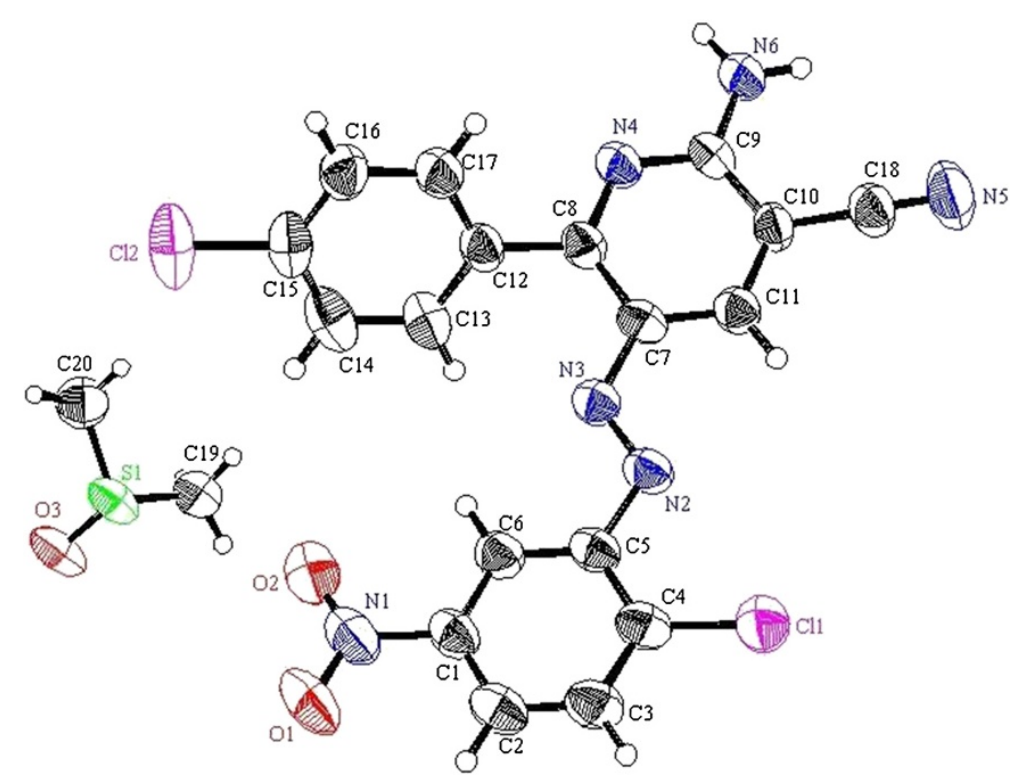

Figure 5 ORTEP plot of the X-ray crystallographic data determined for $8 \mathrm{~h}$.

collected at room temperature. The structure was solved by direct methods and was expanded using Fourier techniques. The non-hydrogen atoms were refined anisotropically. In the case of compounds $8 \mathrm{a}, 8 \mathrm{c}, 8 \mathrm{~h}, 11 \mathrm{a}$ and 12 , all calculations were performed using the Crystal Structure [34] crystallographic software package except for refinement, which was performed using SHELXL-97 [35]. In the case of $\mathbf{2 a}, \mathbf{2 h}, \mathbf{8 b}$, and $10 \mathbf{a}$ the structure was solved and refined using the Bruker SHELXTL Software Package (Structure solution program- SHELXS-97 and Refinement program- SHELXL-97) [35] (cf. Additional files 1, 2, 3, 4, $5,6,7,8,9$ and Table 6). Data were corrected for the absorption effects using the multi-scan method (SADABS). The enaminones $\mathbf{1 a}, \mathbf{b}$ and the arylhydrazonals $\mathbf{2 a - k}$ were prepared according to the literature procedure $[30,31]$.

General procedure for the preparation 2-amino-5-arylazo-6aryl substituted nicotinates $8 a-k$

Independent mixtures of $\mathbf{2 a - g}(10 \mathrm{mmol})$, active methylenenitrile derivatives $\mathbf{3 a}, \mathbf{b}(10 \mathrm{mmol})$, and ammonium acetate $(2 \mathrm{~g})$ in acetic acid $(20 \mathrm{~mL})$ were stirred

Table 1 Selected bond lengths and bond angles for $\mathbf{8 a}$

\begin{tabular}{lccc}
\hline Bond & Bond length $(\AA)$ & Bond & Bond angle(o) \\
\hline N3-C8 & 1.332 & C8-N3-C9 & 119.4 \\
\hline N3-C9 & 1.363 & N3-C8-C7 & 122.5 \\
\hline N4-C9 & 1.327 & N3-C8-C12 & 114.5 \\
\hline C9-C10 & 1.421 & N3-C9-N4 & 116.3 \\
\hline N1-C1 & 1.427 & N4-C9-C10 & 122.5 \\
\hline N1-N2 & 1.259 & N1-N2-C7 & 116.0 \\
\hline N2-C7 & 1.406 & N2-C7-C8 & 116.1 \\
\hline
\end{tabular}

at reflux for $1-2 \mathrm{~h}$. (the progress of the reactions was monitored by using TLC using 1:1 ethyl acetatepetroleum ether as eluent). The mixtures were cooled and then poured into ice-water. The solids that so formed were collected by filtration and crystallized from the proper solvents to give $\mathbf{8} \mathbf{a}-\mathbf{k}$ as pure products.

\section{2-Amino-6-(4-chlorophenyl)-5-(4-chlorophenylazo) nicotinic acid ethyl ester (8a)}

Recrystallized from an EtOH/dioxane (3:1) mixture as orange crystals, yield: (80\%), m.p. $208-210^{\circ} \mathrm{C}$; IR (KBr): $v / \mathrm{cm}^{-1}$ 3409, $3278\left(\mathrm{NH}_{2}\right), 1699$ (CO ester); ${ }^{1} \mathrm{H}-\mathrm{NMR}$ $\left(\mathrm{DMSO}-d_{6}\right): \delta=1.35\left(\mathrm{t}, 3 \mathrm{H}, J=7.2 \mathrm{~Hz}, \mathrm{CH}_{3} \mathrm{CH}_{2}\right), 4.37$ (q, $2 \mathrm{H}, J=7.2 \mathrm{~Hz}, \mathrm{CH}_{3} \mathrm{CH}_{2}$ ), 7.57-7.63 (m, 4H, Ar- $\mathrm{H}$ ), $7.73(\mathrm{~d}, J=8.4 \mathrm{~Hz}, 2 \mathrm{H}, \mathrm{Ar}-\mathrm{H}), 7.81(\mathrm{~d}, J=8.4 \mathrm{~Hz}, 2 \mathrm{H}$, Ar-H), 7.88, 8.14 (two br, $2 \mathrm{H}, \mathrm{NH}_{2}, \mathrm{D}_{2} \mathrm{O}$ exchangeable) and $8.58 \mathrm{ppm}(\mathrm{s}, 1 \mathrm{H}$, pyridine $\mathrm{H} 4) ;{ }^{13} \mathrm{C}$-NMR (DMSO$\left.d_{6}\right): \delta=14.19\left(\mathrm{CH}_{3}\right), 61.23\left(\mathrm{CH}_{2}\right), 105.42$ (pyridine C3), 124.12, 127.51, 127.71, 129.60, 132.75, 134.42, $135.02,135.93,136.38,150.89,159.79,160.62$ and

Table 2 Selected bond lengths and bond angles for $\mathbf{8 h}$

\begin{tabular}{lccc}
\hline Bond & Bond length(Å) & Bond & Bond angle(o) \\
\hline N4-C8 & 1.344 & C8-N4-C9 & 119.6 \\
\hline N4-C9 & 1.352 & N4-C9-C10 & 120.8 \\
\hline N6-C9 & 1.342 & N4-C8-C7 & 121.3 \\
\hline C9-C10 & 1.422 & N4-C9-N6 & 116.9 \\
\hline C10-C18 & 1.433 & N6-C9-C10 & 122.3 \\
\hline N5-C18 & 1.148 & C9-C10-C18 & 119.6 \\
\hline N2-N3 & 1.254 & N5-C18-C10 & 179.2 \\
\hline N3-C7 & 1.412 & N2-N3-C7 & 115.2 \\
\hline
\end{tabular}




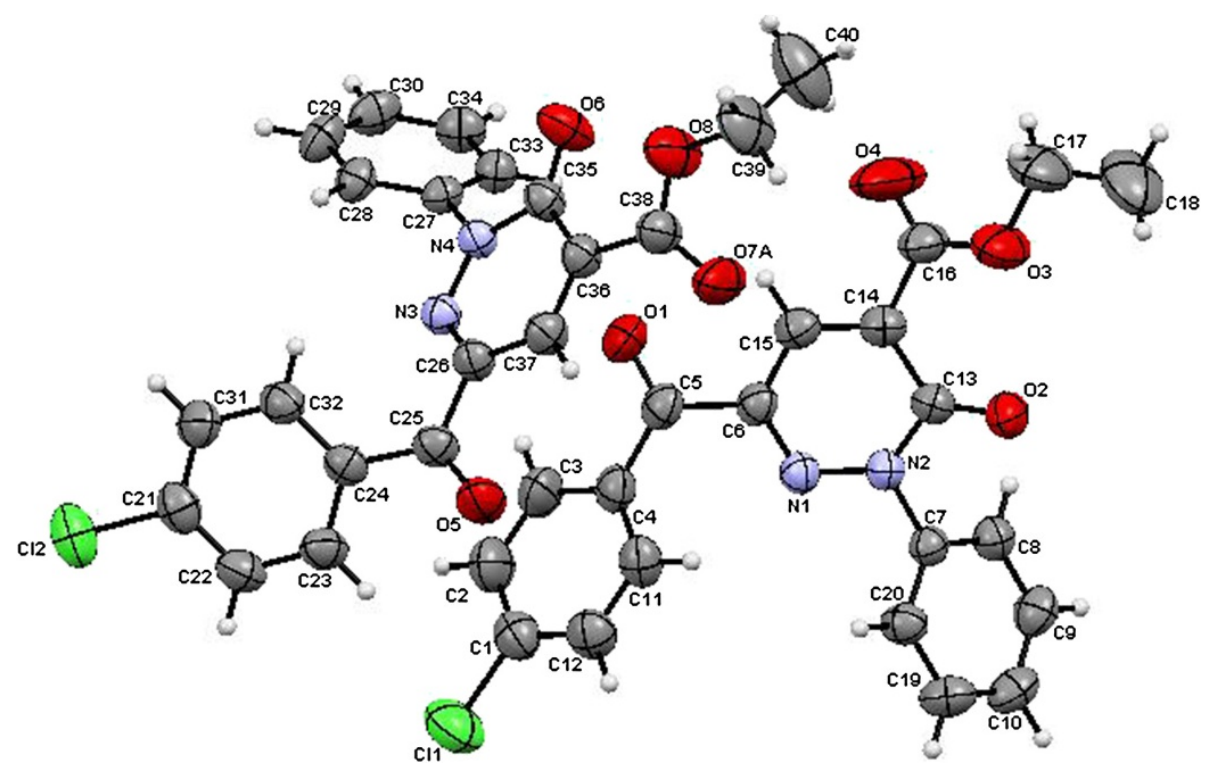

Figure 6 ORTEP plot of the X-ray crystallographic data determined for 10a.

$166.13 \mathrm{ppm}$ (Ar-C and CO); MS (EI): $\mathrm{m} / \mathrm{z}(\%) 414\left(\mathrm{M}^{+}\right.$, 100), $415\left(\mathrm{M}^{+}+1,70.85\right)$. Anal. calcd. for $\mathrm{C}_{20} \mathrm{H}_{16} \mathrm{Cl}_{2} \mathrm{~N}_{4} \mathrm{O}_{2}$ (415.28): C, 57.85; H, 3.88; N, 13.49. Found: C, 57.93; H, $3.77 ; \mathrm{N}, 13.57$.

\section{2-Amino-6-(4-chlorophenyl)-5-(4-nitrophenylazo)nicotinic acid ethyl ester (8b)}

Recrystallized from an EtOH/dioxane (2:1) mixture as deep orange crystals, yield: (85\%), m.p. $230-231^{\circ} \mathrm{C}$; IR $(\mathrm{KBr}): v / \mathrm{cm}^{-1} 3402,3297\left(\mathrm{NH}_{2}\right), 1717$ (CO ester); ${ }^{1} \mathrm{H}-$ NMR (DMSO- $\left.d_{6}\right): \delta=1.36\left(\mathrm{t}, 3 \mathrm{H}, J=7.2 \mathrm{~Hz}, \mathrm{CH}_{3} \mathrm{CH}_{2}\right.$ ), 4.36 (q, $\left.2 \mathrm{H}, J=7.2 \mathrm{~Hz}, \mathrm{CH}_{3} \mathrm{CH}_{2}\right), 7.58$ (d, $J=8.4 \mathrm{~Hz}, 2 \mathrm{H}$, Ar-H), 7.80-7.86 (m, 4H, Ar-H), 7.98, 8.31 (two br, 2H, $\mathrm{NH}_{2}, \mathrm{D}_{2} \mathrm{O}$ exchangeable), $8.35(\mathrm{~d}, J=8.4 \mathrm{~Hz}, 2 \mathrm{H}, \mathrm{Ar}-\mathrm{H})$ and $8.57 \mathrm{ppm}$ (s, $1 \mathrm{H}$, pyridine $\mathrm{H} 4) ;{ }^{13} \mathrm{C}-\mathrm{NMR}$ (DMSO- $d_{6}$ ): $\delta=14.11\left(\mathrm{CH}_{3}\right), 61.25\left(\mathrm{CH}_{2}\right), 105.60$ (pyridine $\mathrm{C} 3$ ), $123.24,125.03,127.46,127.70,132.76,134.58,135.68$, $136.65,147.58,155.58,160.15,161.64$ and $165.94 \mathrm{ppm}$ (Ar-C and CO); MS (EI): m/z (\%) $425\left(\mathrm{M}^{+}, 100\right), 426$ $\left(\mathrm{M}^{+}+1\right.$, 57.92). Anal. calcd. for $\mathrm{C}_{20} \mathrm{H}_{16} \mathrm{ClN}_{5} \mathrm{O}_{4}$ (425.83): C, 56.41; H, 3.79; N, 16.45. Found: C, 56.50; H, 3.72; N, 16.40 .

Table 3 Selected bond lengths and bond angles for 10a

\begin{tabular}{lccc}
\hline Bond & Bond length( $(\AA)$ & Bond & Bond angle(o) \\
\hline N1-N2 & 1.346 & N1-N2-C13 & 126.04 \\
\hline N1-C6 & 1.312 & N1-N2-C7 & 114.44 \\
\hline N2-C13 & 1.413 & C13-N2-C7 & 119.32 \\
\hline O2-C13 & 1.217 & N2-C13-C14 & 112.98 \\
\hline O1-C5 & 1.220 & C6-N1-N2 & 117.37 \\
\hline N2-C7 & 1.442 & O3-C16-C14 & 116.3 \\
\hline
\end{tabular}

2-Amino-6-(4-chlorophenyl)-5-(3-chlorophenylazo)nicotinic acid ethyl ester (8c)

Recrystallized from acetic acid as orange crystals, yield: (76\%), m.p. $188-190^{\circ} \mathrm{C}$; IR (KBr): $v / \mathrm{cm}^{-1} 3400,3275$ $\left(\mathrm{NH}_{2}\right), 1688$ (CO ester); ${ }^{1} \mathrm{H}-\mathrm{NMR}\left(\mathrm{DMSO}-d_{6}\right): \delta=1.35$ $\left(\mathrm{t}, 3 \mathrm{H}, J=7.2 \mathrm{~Hz}, \mathrm{CH}_{3} \mathrm{CH}_{2}\right), 4.35(\mathrm{q}, 2 \mathrm{H}, J=7.2 \mathrm{~Hz}$, $\left.\mathrm{CH}_{3} \mathrm{CH}_{2}\right), 7.52-7.57(\mathrm{~m}, 4 \mathrm{H}, \mathrm{Ar}-\mathrm{H}), 7.61-7.66(\mathrm{~m}, 2 \mathrm{H}$, Ar-H), 7.78 (d, $J=8.4 \mathrm{~Hz}, 2 \mathrm{H}, \mathrm{Ar}-\mathrm{H}$ ), 7.87, 8.15 (two br, $2 \mathrm{H}, \mathrm{NH}_{2}, \mathrm{D}_{2} \mathrm{O}$ exchangeable) and $8.51 \mathrm{ppm}(\mathrm{s}, 1 \mathrm{H}$, pyridine $\mathrm{H} 4) ;{ }^{13} \mathrm{C}-\mathrm{NMR}$ (DMSO- $\left.d_{6}\right): \delta=14.60\left(\mathrm{CH}_{3}\right)$, $61.65\left(\mathrm{CH}_{2}\right), 105.85$ (pyridine $\mathrm{C} 3$ ), 121.99, 122.14, 127.90, $128.03,130.44,131.57,133.20,134.48,134.91,136.31$, 136.66, 153.70, 160.34, 161.26 and $166.51 \mathrm{ppm}$ (Ar-C and CO); MS (EI): m/z (\%) $414\left(\mathrm{M}^{+}, 100\right), 415\left(\mathrm{M}^{+}+1\right.$, 67.45). Anal. calcd. for $\mathrm{C}_{20} \mathrm{H}_{16} \mathrm{Cl}_{2} \mathrm{~N}_{4} \mathrm{O}_{2}$ (415.28): C, 57.85; H, 3.88; N, 13.49. Found: C, 57.78; H, 3.94; N, 13.42.

\section{2-Amino-5-(3-bromophenylazo)-6-(4-chlorophenyl) nicotinic acid ethyl ester (8d)}

Recrystallized from ethanol as deep yellow crystals, yield: (73\%), m.p. $180-181^{\circ} \mathrm{C}$; IR (KBr): $v / \mathrm{cm}^{-1} 3420,3286$ $\left(\mathrm{NH}_{2}\right), 1697$ (CO ester); ${ }^{1} \mathrm{H}-\mathrm{NMR}$ (DMSO- $\left.d_{6}\right): \delta=1.35$ $\left(\mathrm{t}, 3 \mathrm{H}, J=7.2 \mathrm{~Hz}, \mathrm{CH}_{3} \mathrm{CH}_{2}\right), 4.35(\mathrm{q}, 2 \mathrm{H}, J=7.2 \mathrm{~Hz}$, $\mathrm{CH}_{3} \mathrm{CH}_{2}$ ), 7.48 (t, $\left.J=8.0 \mathrm{~Hz}, 1 \mathrm{H}, \mathrm{Ar}-\mathrm{H}\right), 7.55$ (d, $J=8.4$ $\mathrm{Hz}, 2 \mathrm{H}, \mathrm{Ar}-\mathrm{H}), 7.64-7.70(\mathrm{~m}, 2 \mathrm{H}, \mathrm{Ar}-\mathrm{H}), 7.75-7.80$ (m, 3H, Ar-H), 7.88, 8.15 (two br, $2 \mathrm{H}, \mathrm{NH}_{2}, \mathrm{D}_{2} \mathrm{O}$ exchangeable) and $8.51 \mathrm{ppm}(\mathrm{s}, 1 \mathrm{H}$, pyridine $\mathrm{H} 4) ;{ }^{13} \mathrm{C}-\mathrm{NMR}$ $\left(\right.$ DMSO- $\left.d_{6}\right): \delta=14.19\left(\mathrm{CH}_{3}\right), 61.24\left(\mathrm{CH}_{2}\right), 105.45$ (pyridine C3), 122.19, 122.50, 124.44, 127.52, 127.63, 131.51, $132.80,132.92,134.49,135.88,136.24,153.41,159.93$, 160.94 and $166.09 \mathrm{ppm}$ (Ar-C and CO); MS (EI): m/z (\%) $459\left(\mathrm{M}^{+}, 100\right), 460\left(\mathrm{M}^{+}+1,69.55\right)$. Anal. calcd. for 


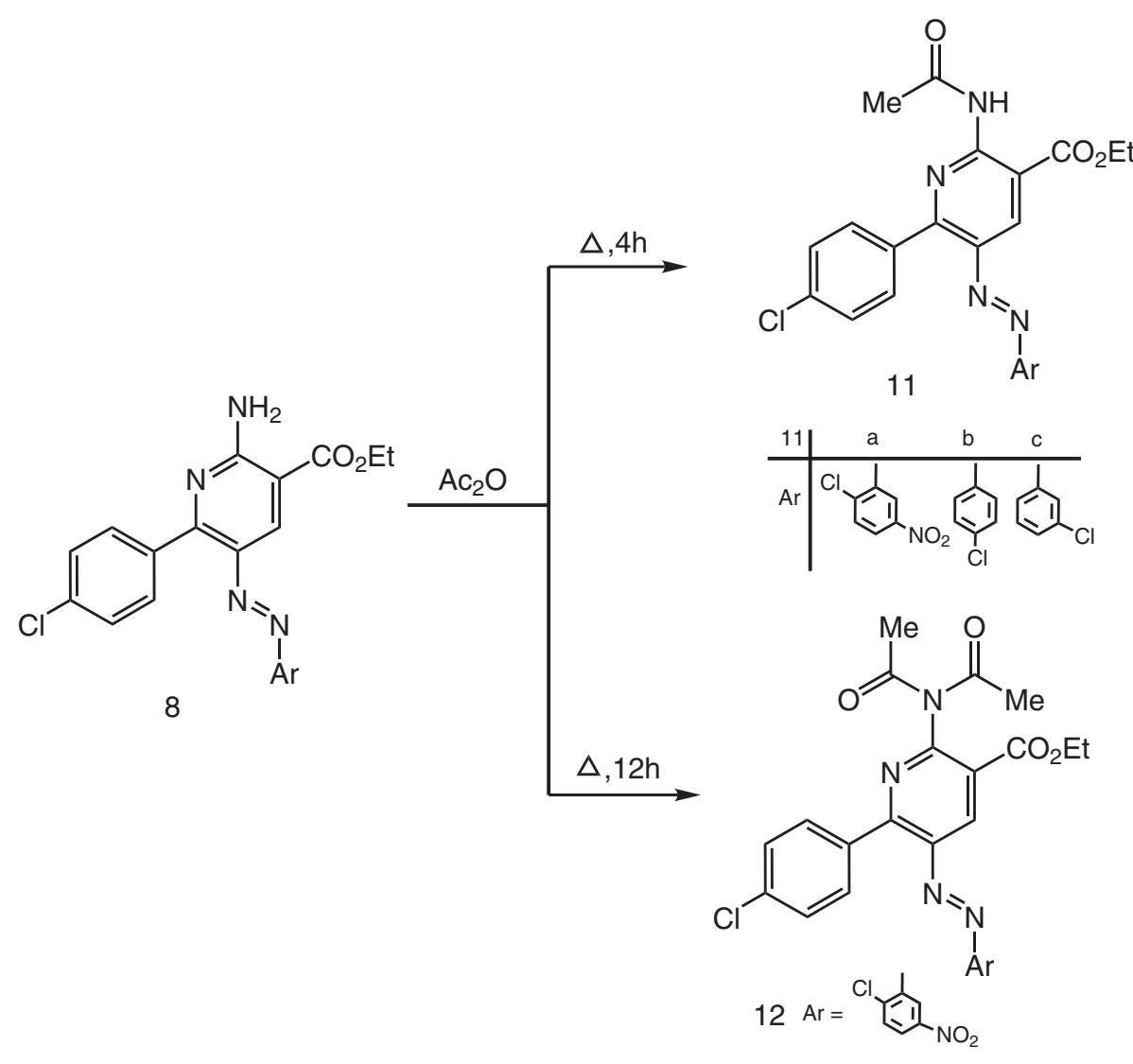

Scheme 2 Synthesis of acylated azonicotinate derivatives 11 and 12.

$\mathrm{C}_{20} \mathrm{H}_{16} \mathrm{BrClN}_{4} \mathrm{O}_{2}$ (459.73): C, 52.25; H, 3.51; N, 12.19 . Found: C, 52.33; H, 3.45; N, 12.23 .

\section{2-Amino-6-(4-chlorophenyl)-5-(2-nitrophenylazo)nicotinic acid ethyl ester (8e)}

Recrystallized from an dioxane mixture as orange crystals, yield: (88\%), m.p. $221-222^{\circ} \mathrm{C}$; IR (KBr): $v / \mathrm{cm}^{-1} 3394,3280$ $\left(\mathrm{NH}_{2}\right), 1704$ (CO ester); ${ }^{1} \mathrm{H}-\mathrm{NMR}\left(\mathrm{DMSO}-d_{6}\right): \delta=1.33$ $\left(\mathrm{t}, 3 \mathrm{H}, J=7.2 \mathrm{~Hz}, \mathrm{CH}_{3} \mathrm{CH}_{2}\right), 4.35(\mathrm{q}, 2 \mathrm{H}, J=7.2 \mathrm{~Hz}$, $\mathrm{CH}_{3} \mathrm{CH}_{2}$ ), 7.50 (d, $\left.J=8.0 \mathrm{~Hz}, 1 \mathrm{H}, \mathrm{Ar}-\mathrm{H}\right), 7.57$ (d, $J=8.4$ $\mathrm{Hz}, 2 \mathrm{H}, \mathrm{Ar}-\mathrm{H}), 7.67$ (t, $J=8.0 \mathrm{~Hz}, 1 \mathrm{H}, \mathrm{Ar}-\mathrm{H}), 7.76-7.82$ (m, 3H, Ar-H), 7.95, 8.31 (two br, $2 \mathrm{H}, \mathrm{NH}_{2}, \mathrm{D}_{2} \mathrm{O}$ exchangeable), $8.05(\mathrm{~d}, J=8.0 \mathrm{~Hz}, 1 \mathrm{H}, \mathrm{Ar}-\mathrm{H})$ and $8.47 \mathrm{ppm}$ (s, $1 \mathrm{H}$, pyridine $\mathrm{H} 4) ;{ }^{13} \mathrm{C}-\mathrm{NMR}$ (DMSO- $\left.d_{6}\right): \delta=14.07$ $\left(\mathrm{CH}_{3}\right), 61.26\left(\mathrm{CH}_{2}\right), 105.69$ (pyridine C3), 118.66, 124.14, $127.78,128.04,130.73,132.66,133.57,134.58,135.68$, 136.81, 144.31, 147.05, 160.12, 161.46 and $165.90 \mathrm{ppm}$ (Ar-C and CO); MS (EI): m/z (\%) $425\left(\mathrm{M}^{+}, 17.25\right), 426$ $\left(\mathrm{M}^{+}+1,7.05\right)$. HRMS (EI): $\mathrm{m} / \mathrm{z}$ calcd. for $\mathrm{C}_{20} \mathrm{H}_{16}^{35} \mathrm{ClN}_{5} \mathrm{O}_{4}$ $\left(\mathrm{M}^{+}\right)$425.0885, found 425.0881.

\section{2-Amino-5-(2-chloro-5-nitrophenylazo)-6-(4-chlorophenyl)} nicotinic acid ethyl ester (8f)

Recryst- allized from DMF as deep orange crystals, yield: (89\%), m.p. $266-268^{\circ} \mathrm{C}$; IR (KBr): $v / \mathrm{cm}^{-1} 3378,3281$ $\left(\mathrm{NH}_{2}\right), 1709$ (CO ester); ${ }^{1} \mathrm{H}-\mathrm{NMR}\left(\mathrm{DMSO}-d_{6}\right): \delta=1.35$ $\left(\mathrm{t}, 3 \mathrm{H}, J=7.2 \mathrm{~Hz}, \mathrm{CH}_{3} \mathrm{CH}_{2}\right), 4.38(\mathrm{q}, 2 \mathrm{H}, J=7.2 \mathrm{~Hz}$, $\left.\mathrm{CH}_{3} \mathrm{CH}_{2}\right), 7.57$ (d, $\left.J=8.4 \mathrm{~Hz}, 2 \mathrm{H}, \mathrm{Ar}-\mathrm{H}\right), 7.85(\mathrm{~d}, J=8.4$ $\mathrm{Hz}, 2 \mathrm{H}, \mathrm{Ar}-\mathrm{H}), 7.96-8.41\left(\mathrm{~m}, 5 \mathrm{H}, 3 \mathrm{Ar}-\mathrm{H}\right.$ and $\left.\mathrm{NH}_{2}\right)$ and $8.63 \mathrm{ppm}$ (s, $1 \mathrm{H}$, pyridine $\mathrm{H} 4$ ); ${ }^{13} \mathrm{C}-\mathrm{NMR}$ (DMSO- $d_{6}$, at $\left.100^{\circ} \mathrm{C}\right): \delta=13.95\left(\mathrm{CH}_{3} \mathrm{CH}_{2}\right), 61.24\left(\mathrm{CH}_{3} \mathrm{CH}_{2}\right), 106.27$ (pyridine C3), 112.67, 124.64, 127.54, 128.12, 131.94, $132.63,134.75,135.81,137.06,139.12,147.22$, 148.64, 160.30, 161.67 and $165.88 \mathrm{ppm}$ (Ar-C and CO); MS (EI): $\mathrm{m} / \mathrm{z}(\%) 459\left(\mathrm{M}^{+}, 100\right), 460\left(\mathrm{M}^{+}+1,71.22\right)$. Anal. calcd. for $\mathrm{C}_{20} \mathrm{H}_{15} \mathrm{Cl}_{2} \mathrm{~N}_{5} \mathrm{O}_{4}$ (460.28): C, 52.19; $\mathrm{H}, 3.28 ; \mathrm{N}, 15.22$. Found: C, 52.23; H, 3.35; N, 15.19 .

\section{2-Amino-5-(4-chlorophenylazo)-6-(4-nitrophenyl)nicotinic acid ethyl ester (8g)}

Recrystallized from an EtOH/dioxane (2:1) mixture as orange crystals, yield: (74\%), m.p. $205-206^{\circ} \mathrm{C}$; IR (KBr): 


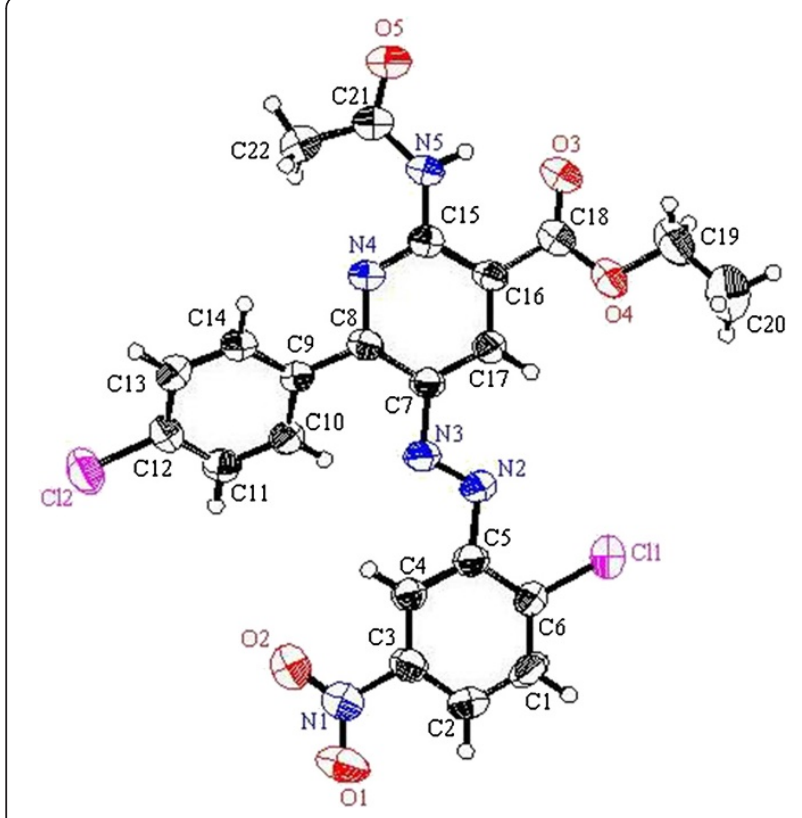

Figure 7 ORTEP plot of the X-ray crystallographic data determined for 11 a.

$v / \mathrm{cm}^{-1}$ 3410, $3311\left(\mathrm{NH}_{2}\right), 1723$ (CO ester); ${ }^{1} \mathrm{H}-\mathrm{NMR}$ (DMSO- $\left.d_{6}\right): \delta=1.37\left(\mathrm{t}, 3 \mathrm{H}, J=7.2 \mathrm{~Hz}, \mathrm{CH}_{3} \mathrm{CH}_{2}\right.$ ), 4.39 (q, $\left.2 \mathrm{H}, J=7.2 \mathrm{~Hz}, \mathrm{CH}_{3} \mathrm{CH}_{2}\right), 7.61(\mathrm{~d}, J=8.4 \mathrm{~Hz}, 2 \mathrm{H}, \mathrm{Ar}-\mathrm{H})$, $7.73(\mathrm{~d}, J=8.4 \mathrm{~Hz}, 2 \mathrm{H}, \mathrm{Ar}-\mathrm{H}), 8.05(\mathrm{~d}, J=8.4 \mathrm{~Hz}, 2 \mathrm{H}$, Ar- $\mathrm{H}$ ), 7.92, 8.20 (two br, $2 \mathrm{H}, \mathrm{NH}_{2}, \mathrm{D}_{2} \mathrm{O}$ exchangeable), $8.37(\mathrm{~d}, J=8.4 \mathrm{~Hz}, 2 \mathrm{H}, \mathrm{Ar}-\mathrm{H})$ and $8.62 \mathrm{ppm}(\mathrm{s}, 1 \mathrm{H}$, pyridine $\mathrm{H} 4) ;{ }^{13} \mathrm{C}$-NMR (DMSO- $\left.d_{6}\right): \delta=14.62\left(\mathrm{CH}_{3}\right), 61.78$ $\left(\mathrm{CH}_{2}\right), 106.68$ (pyridine $\mathrm{C} 3$ ), 123.16, 124.65, 128.07, 130.04, 132.60, 135.64, 137.06, 143.94, 148.19, 151.27, 157.18, 160.23 and $166.44 \mathrm{ppm}$ (Ar-C and CO); MS (EI): $\mathrm{m} / \mathrm{z}(\%) 425\left(\mathrm{M}^{+}, 100\right), 426\left(\mathrm{M}^{+}+1,85.15\right)$. Anal.

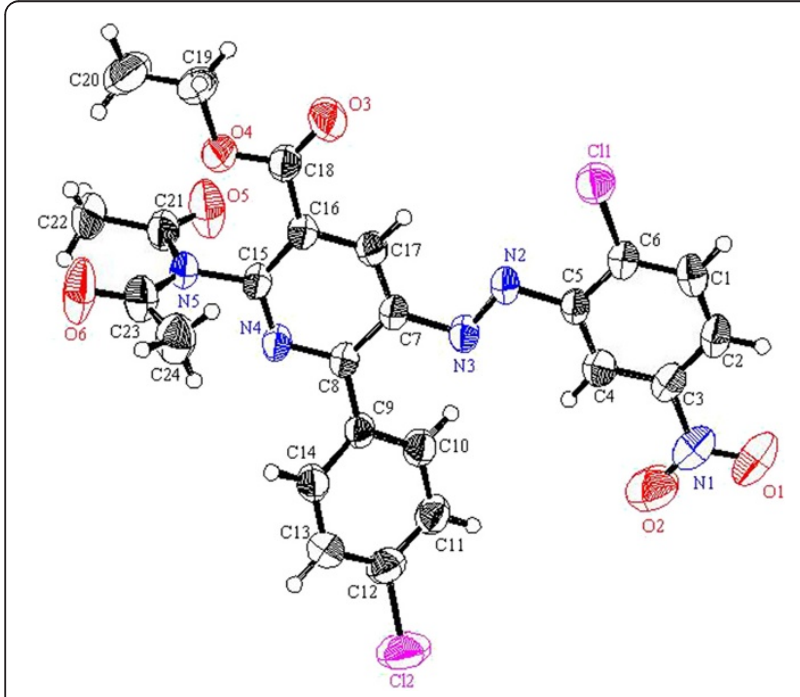

Figure 8 ORTEP plot of the X-ray crystallographic data determined for 12 . calcd. for $\mathrm{C}_{20} \mathrm{H}_{16} \mathrm{ClN}_{5} \mathrm{O}_{4}$ (425.83): C, 56.41; $\mathrm{H}, 3.79 ; \mathrm{N}$, 16.45. Found: C, 56.48; H, 3.85; N, 16.52.

\section{2-Amino-5-(2-chloro-5-nitrophenylazo)-6-(4-chlorophenyl) nicotinonitrile (8h)}

Recrystallized from DMSO as reddish brown crystals, yield: (77\%), m.p. above $300^{\circ} \mathrm{C}$; IR $(\mathrm{KBr}): v / \mathrm{cm}^{-1} 3489$, $3379\left(\mathrm{NH}_{2}\right), 2220(\mathrm{CN}), 1628(\mathrm{C}=\mathrm{N}) ;{ }^{1} \mathrm{H}-\mathrm{NMR}\left(\mathrm{DMSO}-d_{6}\right)$ : $\delta=7.57(\mathrm{~d}, J=8.4 \mathrm{~Hz}, 2 \mathrm{H}, \mathrm{Ar}-\mathrm{H}), 7.81(\mathrm{~d}, J=8.4 \mathrm{~Hz}, 2 \mathrm{H}$, Ar-H), 7.96 (d, $J=8.8 \mathrm{~Hz}, 1 \mathrm{H}, \mathrm{Ar}-\mathrm{H}), 8.10(\mathrm{~s}, 1 \mathrm{H}, \mathrm{Ar}-\mathrm{H})$, 8.17 (br, $2 \mathrm{H}, \mathrm{NH}_{2}, \mathrm{D}_{2} \mathrm{O}$ exchangeable), 8.27 (d, $J=8.8 \mathrm{~Hz}$, $1 \mathrm{H}, \mathrm{Ar}-\mathrm{H})$ and $8.36 \mathrm{ppm}(\mathrm{s}, 1 \mathrm{H}$, pyridine $\mathrm{H} 4) ;{ }^{13} \mathrm{C}-\mathrm{NMR}$ $\left(\right.$ DMSO- $\left.d_{6}\right): \delta=90.85$ (pyridine C3), 112.58, 115.83, $125.24,127.69,131.14,132.13,132.81,134.92,135.42$, 136.15, 139.71, 146.97, 148.04, 160.51 and $161.67 \mathrm{ppm}$ (CN and Ar-C); MS (EI): m/z (\%) $412\left(\mathrm{M}^{+}, 100\right), 413$ $\left(\mathrm{M}^{+}+1\right.$, 61.45). Anal. calcd. for $\mathrm{C}_{18} \mathrm{H}_{10} \mathrm{Cl}_{2} \mathrm{~N}_{6} \mathrm{O}_{2}$ (413.23): C, 52.32; H, 2.44; N, 20.34. Found: C, 52.25; H, 2.53; N, 20.40 .

\section{2-Amino-6-(4-chlorophenyl)-5-(4-chlorophenylazo) nicotinonitrile (8i)}

Recrystallized from dioxane as brown crystals, yield: (69\%), m.p. $278-280^{\circ} \mathrm{C}$; IR (KBr): $v / \mathrm{cm}^{-1} 3441,3338$ $\left(\mathrm{NH}_{2}\right), 2213(\mathrm{CN}), 1656(\mathrm{C}=\mathrm{N}) ;{ }^{1} \mathrm{H}-\mathrm{NMR}\left(\mathrm{DMSO}-d_{6}\right): \delta=$ 7.53-7.59 (m, 4H, Ar-H), 7.79 (d, $J=8.4 \mathrm{~Hz}, 2 \mathrm{H}, \mathrm{Ar}-\mathrm{H})$, $7.84(\mathrm{~d}, J=8.4 \mathrm{~Hz}, 2 \mathrm{H}, \mathrm{Ar}-\mathrm{H}), 8.10$ (br, $2 \mathrm{H}, \mathrm{NH}_{2}, \mathrm{D}_{2} \mathrm{O}$ exchangeable) and 8.34 ppm (s, $1 \mathrm{H}$, pyridine $\mathrm{H} 4)$; MS (EI): $\mathrm{m} / \mathrm{z}(\%) 367\left(\mathrm{M}^{+}, 74.75\right), 368\left(\mathrm{M}^{+}+1,29.50\right)$. Anal. calcd. for $\mathrm{C}_{18} \mathrm{H}_{11} \mathrm{Cl}_{2} \mathrm{~N}_{5}$ (368.23): C, 58.71; $\mathrm{H}, 3.01 ; \mathrm{N}, 19.02$. Found: C, 58.62; H, 2.95; N, 19.11 .

\section{2-Amino-5-(3-bromophenylazo)-6-(4-chlorophenyl) nicotinonitrile (8j)}

Recrystallized from dioxane as brown crystals, yield: (72\%), m.p. above $300^{\circ} \mathrm{C}$; IR (KBr): $v / \mathrm{cm}^{-1} 3424,3317$ $\left(\mathrm{NH}_{2}\right), 2208(\mathrm{CN}), 1639(\mathrm{C}=\mathrm{N}) ;{ }^{1} \mathrm{H}-\mathrm{NMR}\left(\mathrm{DMSO}-d_{6}\right)$ : $\delta=7.56-7.63(\mathrm{~m}, 5 \mathrm{H}, \mathrm{Ar}-\mathrm{H}), 7.77-7.91(\mathrm{~m}, 3 \mathrm{H}, 1 \mathrm{Ar}-\mathrm{H}$ and $\left.\mathrm{NH}_{2}\right), 7.97(\mathrm{~d}, J=8.4 \mathrm{~Hz}, 2 \mathrm{H}, \mathrm{Ar}-\mathrm{H})$ and $8.22 \mathrm{ppm}$ (s, $1 \mathrm{H}$, pyridine $\mathrm{H} 4)$; $\mathrm{MS}(\mathrm{EI}): \mathrm{m} / \mathrm{z}(\%) 412\left(\mathrm{M}^{+}, 100\right)$, $413\left(\mathrm{M}^{+}+1\right.$, 31.26). Anal. calcd. for $\mathrm{C}_{18} \mathrm{H}_{11} \mathrm{BrClN}_{5}$ (412.68): C, 52.39; H, 2.69; N, 16.97. Found: C, 52.47; H, $2.75 ; \mathrm{N}, 17.05$.

\section{2-Amino-6-(4-chlorophenyl)-5-(2-nitrophenylazo) nicotinonitrile (8k)}

Recrystallized from a DMF/dioxane (1:1) mixture as yellowish brown crystals: (70\%), m.p. $275-276^{\circ} \mathrm{C}$; IR $(\mathrm{KBr}): \quad v / \mathrm{cm}^{-1}$ 3477, $3367\left(\mathrm{NH}_{2}\right), 2222(\mathrm{CN}), 1635$ $(\mathrm{C}=\mathrm{N}) ;{ }^{1} \mathrm{H}-\mathrm{NMR}\left(\mathrm{DMSO}-d_{6}\right): \delta=7.48(\mathrm{~d}, J=7.6 \mathrm{~Hz}$, $1 \mathrm{H}$, Ar-H), $7.57(\mathrm{~d}, J=8.0 \mathrm{~Hz}, 2 \mathrm{H}$, Ar- $\mathrm{H}), 7.67(\mathrm{t}, J=7.6$ $\mathrm{Hz}, 1 \mathrm{H}, \mathrm{Ar}-\mathrm{H}), 7.77-7.79(\mathrm{~m}, 3 \mathrm{H}, \mathrm{Ar}-\mathrm{H}), 8.04-8.10$ ( $\mathrm{m}, 3 \mathrm{H}, 1 \mathrm{Ar}-\mathrm{H}$ and $\mathrm{NH}_{2}, \mathrm{D}_{2} \mathrm{O}$ exchangeable), $8.16 \mathrm{ppm}$ 


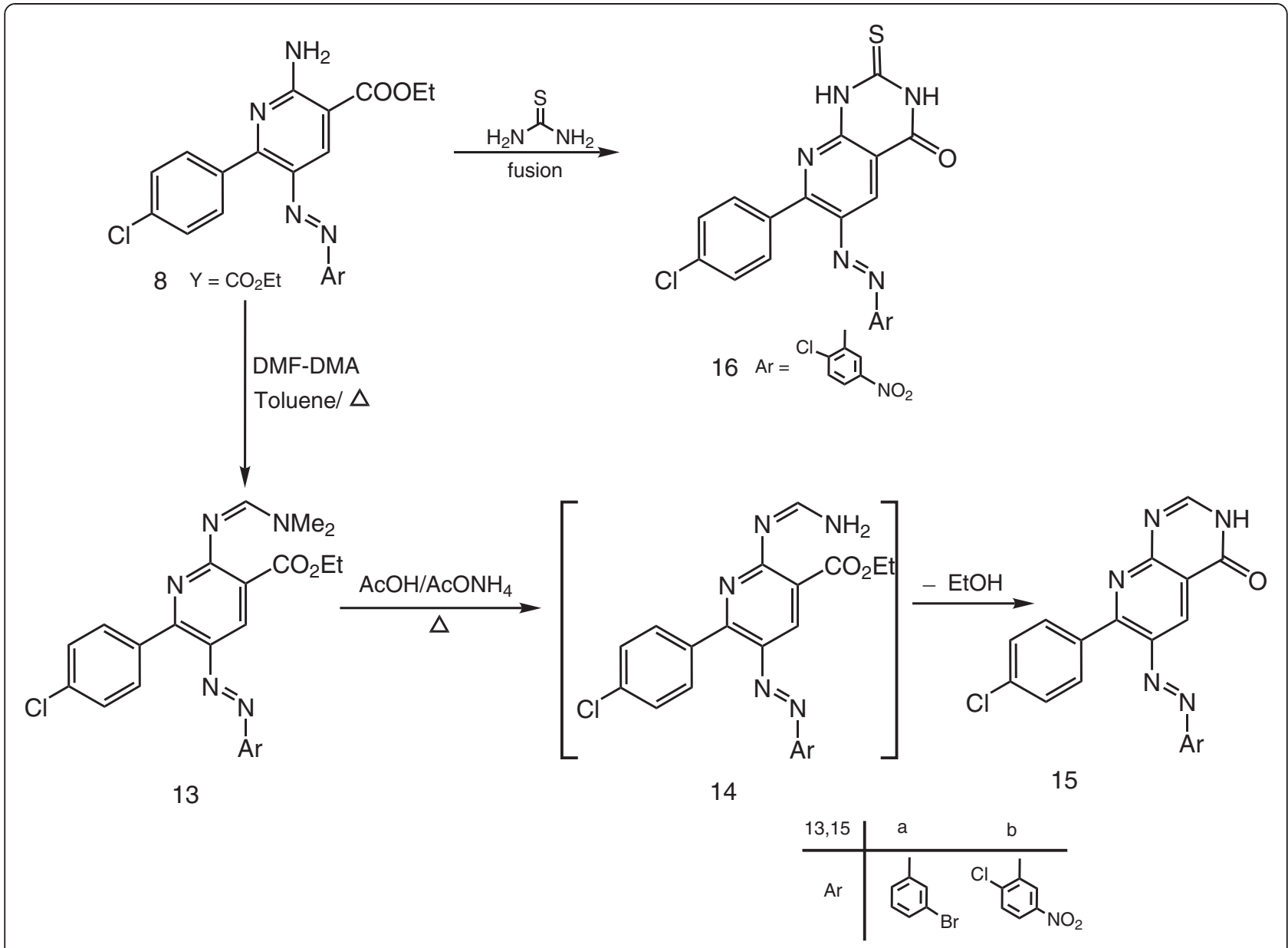

Scheme 3 Synthesis of pyrido[2,3-d]pyrimidine derivatives 15 and 16.

(s, $1 \mathrm{H}$, pyridine $\mathrm{H} 4$ ); ${ }^{13} \mathrm{C}$-NMR (DMSO- $d_{6}$ ): $\delta=90.62$ (pyridine C3), 115.89, 118.52, 124.17, 127.83, 131.02, 131.20, 132.65, 133.59, 134.79, 135.44, 136.42, 144.07, 147.18, 160.30 and 161.14 ppm (Ar-C and CN); MS (EI): $\mathrm{m} / \mathrm{z}(\%) 478\left(\mathrm{M}^{+}, 100\right), 426\left(\mathrm{M}^{+}+1,46.55\right)$. HRMS (EI): $\mathrm{m} / \mathrm{z}$ calcd. for $\mathrm{C}_{18} \mathrm{H}_{11}^{35} \mathrm{ClN}_{6} \mathrm{O}_{2}\left(\mathrm{M}^{+}\right)$378.0626, found 378.0627 .

\section{General procedure for the preparation pyridazine derivatives 10a-d}

Independent mixtures of $\mathbf{2 h}-\mathbf{k}(10 \mathrm{mmol})$, ethyl cyanoacetate 3a (1.15 g, $10 \mathrm{mmol})$, and ammonium acetate $(2 \mathrm{~g})$ in acetic acid $(20 \mathrm{~mL})$ were stirred at reflux for $1-2 \mathrm{~h}$. (the progress of the reactions was monitored by using TLC using 1:1 ethyl acetate-petroleum ether as eluent). The mixtures were cooled and then poured into iced water. The solids that so formed were collected by filtration and recrystallized from the proper solvents to give 10a-d as pure products.
6-(4-Chlorobenzoyl)-3-oxo-2-phenyl-2,3-dihydropyridazine4-carboxylic acid ethyl ester (10a)

Recrystallized from an EtOH as yellow crystals, yield: (75\%), m.p. $140-141^{\circ} \mathrm{C}$; IR (KBr): $v / \mathrm{cm}^{-1} 1715,1690$ (CO and CO ester); ${ }^{1} \mathrm{H}-\mathrm{NMR}$ (DMSO- $\left.d_{6}\right): \delta=1.32(\mathrm{t}, 3 \mathrm{H}$, $\left.J=7.2 \mathrm{~Hz}, \mathrm{CH}_{3} \mathrm{CH}_{2}\right), 4.35\left(\mathrm{q}, 2 \mathrm{H}, J=7.2 \mathrm{~Hz}, \mathrm{CH}_{3} \mathrm{CH}_{2}\right)$, 7.46-7.55 (m, 3H, Ar-H), 7.60-7.62 (m, 4H, Ar-H), 8.04 $(\mathrm{d}, J=8.4 \mathrm{~Hz}, 2 \mathrm{H}, \mathrm{Ar}-\mathrm{H})$, and $8.33 \mathrm{ppm}(\mathrm{s}, 1 \mathrm{H}$, pyridazine H5); ${ }^{13} \mathrm{C}-\mathrm{NMR}$ (DMSO- $\left.d_{6}\right): \delta=13.97\left(\mathrm{CH}_{3}\right), 61.86\left(\mathrm{CH}_{2}\right)$, $125.94,128.48,128.87,128.95,131.06,131.93,132.41$, $133.75,138.38,141.04,141.27,155.92,162.60$, and 187.71 ppm (Ar-C and CO); MS (EI): m/z (\%) $382\left(\mathrm{M}^{+}, 100\right)$, $383\left(\mathrm{M}^{+}+1\right.$, 32.85). Anal. calcd. for $\mathrm{C}_{20} \mathrm{H}_{15} \mathrm{ClN}_{2} \mathrm{O}_{4}$ (382.81): C, 62.75; H, 3.95; N, 7.32. Found: C, 62.82; H, 3.92; N, 7.28.

6-(4-Chlorobenzoyl)-3-oxo-2-p-tolyl-2,3-dihydropyridazine-4carboxylic acid ethyl ester (10b)

Recrystallized from EtOH as yellow crystals, yield: (69\%), m.p. 94-95 $\mathrm{C}$; IR (KBr): $v / \mathrm{cm}^{-1} 1753,1687$ (CO and CO 
Table 4 Inhibition zone diameter $(\mathrm{mm})$ of the tested chemicals that showed antimicrobial activities against the tested microorganisms

\section{Compound No.}

Inhibition zone diameter in

$\mathrm{mm} \pm$ (standard deviation)

\begin{tabular}{|c|c|c|c|c|c|}
\hline & \\
\hline & E. coli & P. aeruginosa & B. subtilis & S. aureus & C. albicans \\
\hline $1 a$ & $20(0.04)$ & 0 & $23(0.02)$ & $12(0.02)$ & $34(0.05)$ \\
\hline $2 a$ & 0 & 0 & $12(0.04)$ & $14(0.10)$ & 0 \\
\hline $2 c$ & 0 & 0 & $12(0.02)$ & $10(0.03)$ & 0 \\
\hline $2 d$ & 0 & 0 & $12(0.03)$ & $10(0.07)$ & 0 \\
\hline $2 e$ & 0 & 0 & $11(0.07)$ & $8(0.07)$ & 0 \\
\hline $2 g$ & 0 & 0 & $16(0.04)$ & $11(0.05)$ & 0 \\
\hline $8 a$ & 0 & 0 & $30(0.07)$ & 0 & 0 \\
\hline $8 b$ & 0 & 0 & $28(0.10)$ & 0 & 0 \\
\hline $8 c$ & 0 & 0 & $23(0.02)$ & 0 & 0 \\
\hline $8 d$ & 0 & 0 & $25(0.09)$ & 0 & 0 \\
\hline $8 e$ & 0 & 0 & $29(0.03)$ & 0 & 0 \\
\hline $8 f$ & 0 & 0 & $27(0.10)$ & 0 & 0 \\
\hline $8 \mathrm{~h}$ & 0 & 0 & $26(0.10)$ & 0 & 0 \\
\hline $10 a$ & 0 & 0 & $22(0.20)$ & 0 & 0 \\
\hline $10 \mathrm{~b}$ & 0 & 0 & $25(0.02)$ & 0 & 0 \\
\hline $10 c$ & 0 & 0 & $29(0.20)$ & 0 & 0 \\
\hline $11 \mathrm{~b}$ & 0 & 0 & $13(0.06)$ & $6(0.06)$ & \\
\hline 12 & 0 & 0 & $11(0.10)$ & $4(0.04)$ & \\
\hline $13 a$ & 0 & 0 & $16(0.05)$ & 0 & 0 \\
\hline $13 b$ & 0 & 0 & $17(0.10)$ & 0 & 0 \\
\hline $15 a$ & 0 & 0 & $19(0.02)$ & $12(0.07)$ & 0 \\
\hline $15 b$ & 0 & 0 & $23(0.03)$ & $9(0.2)$ & 0 \\
\hline 16 & 0 & 0 & $25(0.09)$ & $14(0.02)$ & 0 \\
\hline DMSO (solvent) & 0 & 0 & 0 & 0 & 0 \\
\hline${ }^{a}$ Ampicillin & $23(0.14)$ & $17(0.07)$ & $21(0.05)$ & $26(0.07)$ & 0 \\
\hline${ }^{\mathrm{b}}$ Cycloheximide & - & - & - & - & 0 \\
\hline
\end{tabular}

DMSO $=$ Dimethyl sulfoxide, ${ }^{a}$ Ampicillin antibacterial drug, ${ }^{\mathrm{b}}$ cycloheximide antifungal drug, - not tested.

ester); ${ }^{1} \mathrm{H}$-NMR (DMSO- $d_{6}$ ): $\delta=1.31(\mathrm{t}, 3 \mathrm{H}, J=7.2 \mathrm{~Hz}$, $\left.\mathrm{CH}_{3} \mathrm{CH}_{2}\right), 2.37\left(\mathrm{~s}, 3 \mathrm{H}, \mathrm{CH}_{3}\right), 4.35(\mathrm{q}, 2 \mathrm{H}, J=7.2 \mathrm{~Hz}$, $\mathrm{CH}_{3} \mathrm{CH}_{2}$ ), 7.33 (d, $\left.J=8.4 \mathrm{~Hz}, 2 \mathrm{H}, \mathrm{Ar}-\mathrm{H}\right), 7.49$ (d, $J=8.4$ $\mathrm{Hz}, 2 \mathrm{H}, \mathrm{Ar}-\mathrm{H}), 7.62(\mathrm{~d}, J=8.4 \mathrm{~Hz}, 2 \mathrm{H}, \mathrm{Ar}-\mathrm{H}), 8.03$ (d, $J=8.4 \mathrm{~Hz}, 2 \mathrm{H}, \mathrm{Ar}-\mathrm{H})$ and $8.32 \mathrm{ppm}(\mathrm{s}, 1 \mathrm{H}$, pyridazine H5); ${ }^{13} \mathrm{C}$-NMR (DMSO- $\left.d_{6}\right): \delta=14.43\left(\mathrm{CH}_{3}\right), 21.19$ $\left(\mathrm{CH}_{3}\right), 62.29\left(\mathrm{CH}_{2}\right), 126.13,128.91,129.73,131.41$, $132.22,132.85,134.26,138.75,139.07,139.10,141.62$, 156.38, 163.09 and $188.16 \mathrm{ppm}$ (Ar-C and CO); MS (EI): $\mathrm{m} / \mathrm{z}(\%) 396\left(\mathrm{M}^{+}, 100\right), 397\left(\mathrm{M}^{+}+1,34.52\right)$. Anal. calcd. for $\mathrm{C}_{21} \mathrm{H}_{17} \mathrm{ClN}_{2} \mathrm{O}_{4}$ (396.83): C, 63.56; $\mathrm{H}, 4.32 ; \mathrm{N}, 7.06$. Found: C, 63.63; H, 4.26; N, 7.15.

\section{6-(4-Chlorobenzoyl)-2-(4-methoxyphenyl)-3-oxo-2,3-} dihydropyridazine-4-carboxylic acid ethyl ester (10c)

Recrystallized from EtOH as yellow crystals, yield: (72\%), m.p. $132-133^{\circ} \mathrm{C}$; IR (KBr): $v / \mathrm{cm}^{-1} 1757,1679$ (CO and CO ester); ${ }^{1} \mathrm{H}-\mathrm{NMR}$ (DMSO- $\left.d_{6}\right): \delta=1.32(\mathrm{t}, 3 \mathrm{H}, J=7.2$ $\mathrm{Hz}, \mathrm{CH}_{3} \mathrm{CH}_{2}$ ), 3.81 (s, $\left.3 \mathrm{H}, \mathrm{OCH}_{3}\right), 4.35$ (q, $2 \mathrm{H}, J=7.2$
$\mathrm{Hz}, \mathrm{CH}_{3} \mathrm{CH}_{2}$ ), 7.06 (d, $\left.J=8.8 \mathrm{~Hz}, 2 \mathrm{H}, \mathrm{Ar}-\mathrm{H}\right), 7.53$ (d, $J=$ $8.8 \mathrm{~Hz}, 2 \mathrm{H}, \mathrm{Ar}-\mathrm{H}), 7.62(\mathrm{~d}, J=8.4 \mathrm{~Hz}, 2 \mathrm{H}, \mathrm{Ar}-\mathrm{H}), 8.03$ $(\mathrm{d}, J=8.4 \mathrm{~Hz}, 2 \mathrm{H}, \mathrm{Ar}-\mathrm{H})$ and $8.31 \mathrm{ppm}(\mathrm{s}, 1 \mathrm{H}$, pyridazine H5); ${ }^{13} \mathrm{C}$-NMR (DMSO- $\left.d_{6}\right): \delta=14.43\left(\mathrm{CH}_{3}\right), 55.94\left(\mathrm{CH}_{3}\right)$, $62.28\left(\mathrm{CH}_{2}\right), 114.37,127.63,128.92,131.28,132.15,132.86$, $134.29,134.40,138.74,141.51,156.46,159.75,163.14$ and $188.22 \mathrm{ppm}$ (Ar-C and CO); MS (EI): m/z (\%) $412\left(\mathrm{M}^{+}\right.$, 100), $413\left(\mathrm{M}^{+}+1,28.95\right)$. Anal. calcd. for $\mathrm{C}_{21} \mathrm{H}_{17} \mathrm{ClN}_{2} \mathrm{O}_{5}$ (412.83): C, 61.10; H, 4.15; N, 6.79. Found: C, 61.17; H, $4.21 ; \mathrm{N}, 6.75$.

6-(4-Chlorobenzoyl)-2-(2,3-dimethylphenyl)-3-oxo-2,3dihydropyridazine-4-carboxylic acid ethyl ester (10d)

Recrystallized from EtOH as pale yellow crystals, yield: (70\%), m.p. $96-97^{\circ} \mathrm{C}$; IR (KBr): $v / \mathrm{cm}^{-1} 1746,1692$ (CO and $\mathrm{CO}$ ester); ${ }^{1} \mathrm{H}-\mathrm{NMR}$ (DMSO- $\left.d_{6}\right): \delta=1.33(\mathrm{t}, 3 \mathrm{H}, J=$ $7.2 \mathrm{~Hz}, \mathrm{CH}_{3} \mathrm{CH}_{2}$ ), $2.00\left(\mathrm{~s}, 3 \mathrm{H}, \mathrm{CH}_{3}\right), 2.31\left(\mathrm{~s}, 3 \mathrm{H}, \mathrm{CH}_{3}\right)$, $4.35\left(\mathrm{q}, 2 \mathrm{H}, J=7.2 \mathrm{~Hz}, \mathrm{CH}_{3} \mathrm{CH}_{2}\right), 7.24-7.31(\mathrm{~m}, 3 \mathrm{H}, \mathrm{Ar}-$ $\mathrm{H}), 7.58(\mathrm{~d}, J=8.4 \mathrm{~Hz}, 2 \mathrm{H}, \operatorname{Ar}-\mathrm{H}), 7.95(\mathrm{~d}, J=8.4 \mathrm{~Hz}$, 
Table 5 The MICs $(\mu \mathrm{g} / \mathrm{mL})$ of selected newly synthesized compounds against the tested microorganisms Compound No. The minimum inhibitory concentration

(MIC) in $\mu \mathrm{g} / \mathrm{mL}$

\begin{tabular}{|c|c|c|c|c|c|}
\hline & \\
\hline & E. coli & $P$. aeruginosa & B. subtilis & S. aureus & C. albicans \\
\hline $1 a$ & 50 & - & 50 & - & 12.5 \\
\hline $2 a$ & - & - & 125 & 100 & - \\
\hline $2 g$ & - & - & 100 & - & - \\
\hline $8 a$ & - & - & 12.5 & - & - \\
\hline $8 b$ & - & - & 25 & - & - \\
\hline $8 c$ & - & - & 50 & - & - \\
\hline $8 d$ & - & . & 25 & - & 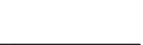 \\
\hline $8 e$ & - & - & 25 & - & - \\
\hline $8 g$ & - & - & 25 & - & - \\
\hline $10 a$ & - & - & 50 & - & - \\
\hline $10 \mathrm{~b}$ & - & - & 25 & - & - \\
\hline $10 c$ & - & - & 12.5 & - & - \\
\hline $11 \mathrm{~b}$ & - & - & 100 & - & - \\
\hline $13 a$ & - & - & 100 & - & - \\
\hline $13 b$ & - & - & 100 & - & - \\
\hline $15 a$ & - & - & 100 & - & - \\
\hline $15 b$ & - & - & 50 & - & - \\
\hline 16 & 0 & 0 & 50 & 100 & - \\
\hline${ }^{a}$ Ampicillin & 6.25 & - & 12.5 & 12.5 & - \\
\hline Cycloheximide & - & - & - & - & - \\
\hline
\end{tabular}

${ }^{a}$ Ampicillin antibacterial drug, ${ }^{\mathrm{b}}$ cycloheximide antifungal drug, - not measured.

Table 6 The crystallographic data for the measured compounds [36]

\begin{tabular}{|c|c|}
\hline Compound No. & Crystal Data \\
\hline $2 \mathrm{a}$ & 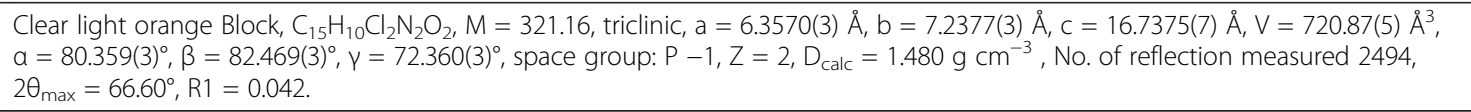 \\
\hline $2 \mathrm{~h}$ & $\begin{array}{l}\text { Clear light yellow Block, } C_{15} \mathrm{H}_{11} \mathrm{CIN}_{2} \mathrm{O}_{2}, \mathrm{M}=286.71 \text {, triclinic, } \mathrm{a}=6.0147(2) \AA, b=7.3767(2) \AA, c=16.5968(4) \AA, V=672.60(3) \AA^{3}, \\
\mathrm{a}=80.941(2)^{\circ}, \beta=85.3620(10)^{\circ}, \gamma=67.6940(10)^{\circ}, \text { space group: } \mathrm{P}-1, \mathrm{Z}=2, \mathrm{D}_{\text {calc }}=1.416 \mathrm{~g} \mathrm{~cm} \mathrm{~cm}^{-3}, \text { No. of reflection measured } 2324 \text {, } \\
2 \theta_{\max }=66.74^{\circ}, \mathrm{R} 1=0.036 .\end{array}$ \\
\hline $8 a$ & $\begin{array}{l}\text { Yellow platelet crystal, } \mathrm{C}_{20} \mathrm{H}_{16} \mathrm{Cl}_{2} \mathrm{~N}_{4} \mathrm{O}_{2}, \mathrm{M}=415.28 \text {, triclinic, } \mathrm{a}=7.796(1) \AA \mathrm{A}, \mathrm{b}=11.004(2) \AA, \mathrm{c}=12.229(2) \AA \mathrm{A}, \mathrm{V}=987.0(3) \AA^{3}, \\
\mathrm{a}=70.789(8)^{\circ}, \beta=89.602(7)^{\circ}, \gamma=85.231(7)^{\circ}, \text { space group: } \mathrm{P}-1, \mathrm{Z}=2, \mathrm{D}_{\text {calc }}=1.397 \mathrm{~g} \mathrm{~cm}{ }^{-3}, \text { No. of reflection measured } 3995, \\
2 \theta_{\max }=52.7^{\circ}, \mathrm{R} 1=0.064 .\end{array}$ \\
\hline $8 b$ & $\begin{array}{l}\text { Clear light orange flake, } \mathrm{C}_{20} \mathrm{H}_{16} \mathrm{ClN} \mathrm{N}_{5} \mathrm{O}_{4}, \mathrm{M}=425.83 \text {, monoclinic, } \mathrm{a}=27.918(4) \AA, \mathrm{b}=6.632(8) \AA, \mathrm{c}=24.125(3) \AA \mathrm{A}, \mathrm{V}=4082.0(9) \AA^{3}, \\
\mathrm{a}=\gamma=90.00^{\circ}, \beta=113.965(9)^{\circ}, \text { space group: } \mathrm{C} 12 / \mathrm{c} 1, \mathrm{Z}=8, \mathrm{D}_{\text {calc }}=1.386 \mathrm{~g} \mathrm{~cm}^{-3}, \text { No. of reflection measured } 3434, \\
2 \theta_{\max }=66.59^{\circ}, \mathrm{R} 1=0.082 .\end{array}$ \\
\hline $8 \mathrm{c}$ & $\begin{array}{l}\text { Orange prism crystal, } \mathrm{C}_{20} \mathrm{H}_{16} \mathrm{Cl}_{2} \mathrm{~N}_{4} \mathrm{O}_{2}, \mathrm{M}=415.28 \text {, orthorhombic, } \mathrm{a}=7.5481(6) \AA, \mathrm{b}=21.382(2) \AA \AA \mathrm{A}, \mathrm{c}=27.862(2) \AA, \mathrm{V}=4496.8(6) \\
\AA^{3}, \mathrm{a}=\beta=\gamma=90.0^{\circ} \text {, space group: Pbca, } \mathrm{Z}=8, \mathrm{D}_{\text {calc }}=1.404 \mathrm{~g} \mathrm{~cm}^{-3}, \text { No. of reflection measured } 3923,2 \theta_{\max }=50.0^{\circ}, \mathrm{R} 1=0.067 .\end{array}$ \\
\hline $8 \mathrm{~h}$ & $\begin{array}{l}\text { Yellow block crystal, } \mathrm{C}_{18} \mathrm{H}_{10} \mathrm{Cl}_{2} \mathrm{~N}_{6} \mathrm{O}_{2}, \mathrm{M}=413.23 \text {, triclinic, } \mathrm{a}=8.918(1) \AA, \mathrm{b}=10.696(1) \AA, \mathrm{A}=13.217(2) \AA, V=1132.2(2) \AA^{3}, a= \\
73.044(6)^{\circ}, \beta=81.609(6)^{\circ}, \mathrm{Y}=70.078(5)^{\circ} \text {, space group: } \mathrm{P}-1, \mathrm{Z}=2, \mathrm{D}_{\text {calc }}=1.441 \mathrm{~g} \mathrm{~cm}^{-3} \text {, No. of reflection measured } 4609,2 \theta_{\max }= \\
52.7^{\circ}, \mathrm{R} 1=0.047 .\end{array}$ \\
\hline 10a & $\begin{array}{l}\text { Clear light colorless block, } \mathrm{C}_{20} \mathrm{H}_{15} \mathrm{ClN}_{2} \mathrm{O}_{4}, \mathrm{M}=382.81, \text { monoclinic, } \mathrm{a}=9.8702(7) \AA, \mathrm{b}=18.7297(14) \AA, \mathrm{c}=19.4912(15) \AA, V=3600.1 \\
(5) \AA^{3}, a=\gamma=90^{\circ}, \beta=92.397(4)^{\circ} \text {, space group: P } 121 / \mathrm{c} 1, Z=8, D_{\text {calc }}=1.412 \mathrm{~g} \mathrm{~cm}{ }^{-3} \text {, No. of reflection measured } 6168,2 \theta_{\max }= \\
66.63^{\circ}, \mathrm{R} 1=0.049 .\end{array}$ \\
\hline 11 & $\begin{array}{l}\text { Yellow needle crystal, } C_{22} \mathrm{H}_{17} \mathrm{Cl}_{2} \mathrm{~N}_{5} \mathrm{O}_{5}, \mathrm{M}=502.32 \text {, orthorhombic, } a=26.252(2) \AA, b=7.3051(5) \AA, c=24.022(2) \AA, V=4606.8(6) \AA^{3}, \\
a=\beta=\gamma=90^{\circ} \text {, space group: } \mathrm{Pbcn}, Z=8, D_{\text {calc }}=1.448 \mathrm{~g} \mathrm{~cm}^{-3} \text {, No. of reflection measured } 4017,2 \theta_{\max }=50.0^{\circ}, R 1=0.059 .\end{array}$ \\
\hline 12 & $\begin{array}{l}\text { Yellow needle crystal, } \mathrm{C}_{24} \mathrm{H}_{1} \mathrm{Cl}_{2} \mathrm{~N}_{5} \mathrm{O}_{6}, \mathrm{M}=544.35, \text { monoclinic, } \mathrm{a}=8.027(1) \AA \mathrm{A}, \mathrm{b}=14.586(2) \AA, \mathrm{c}=21.531(3) \AA, \mathrm{V}=2499.1(5) \AA^{3}, \\
a=y=90^{\circ}, \beta=97.548(7)^{\circ}, \text { space group: } \mathrm{P} 21 / \mathrm{c}, \mathrm{Z}=4, \mathrm{D}_{\text {calc }}=1.447 \mathrm{~g} \mathrm{~cm}^{-3}, \text { No. of reflection measured } 5078,2 \theta_{\max }=52.7^{\circ}, \\
\mathrm{R} 1=0.046 .\end{array}$ \\
\hline
\end{tabular}


$2 \mathrm{H}, \mathrm{Ar}-\mathrm{H})$ and $8.38 \mathrm{ppm}$ (s, 1H, pyridazine $\mathrm{H} 5)$; MS (EI): $\mathrm{m} / \mathrm{z}(\%) 410\left(\mathrm{M}^{+}, 100\right), 411\left(\mathrm{M}^{+}+1,35.14\right)$. Anal. calcd. for $\mathrm{C}_{22} \mathrm{H}_{19} \mathrm{ClN}_{2} \mathrm{O}_{4}(410.86)$ : C, 64.32; H, 4.66; N, 6.82. Found: C, 64.36; H, 4.73; N, 6.89.

\section{General procedure for the preparation compounds $11 a-c$ and 12}

Independent solutions of the azonicotinates 8a,c,f (10 $\mathrm{mmol})$ in acetic anhydride $(10 \mathrm{~mL})$ were stirred at reflux for $4 \mathrm{~h}$. in case of compounds 11a-c and for $12 \mathrm{~h}$. in case of compound 12. Then, the reaction mixture was allowed to cool to room temperature, the formed crude product was collected by filtration washed with ethanol and recrystallized from the proper solvent.

\section{2-Acetylamino-5-(2-chloro-5-nitrophenylazo)-6-(4- chlorophenyl)nicotinic acid ethyl ester (11a)}

Recrystallized from EtOH/dioxane (2:1) mixture as reddish orange crystals, yield: (83\%), m.p. $257-258^{\circ} \mathrm{C}$; IR (KBr): $v / \mathrm{cm}^{-1} 3231(\mathrm{NH}), 1719,1673$ (2 CO); ${ }^{1} \mathrm{H}-\mathrm{NMR}$ $\left(\mathrm{DMSO}-d_{6}\right): \delta=1.30\left(\mathrm{t}, 3 \mathrm{H}, J=7.2 \mathrm{~Hz}, \mathrm{CH}_{3} \mathrm{CH}_{2}\right), 2.23$ (s, 3H, $\mathrm{COCH}_{3}$ ), 4.29 (q, $2 \mathrm{H}, J=7.2 \mathrm{~Hz}, \mathrm{CH}_{3} \mathrm{CH}_{2}$ ), 7.64 (d, $J=8.4 \mathrm{~Hz}, 2 \mathrm{H}, \mathrm{Ar}-\mathrm{H}), 7.91(\mathrm{~d}, J=8.4 \mathrm{~Hz}, 2 \mathrm{H}, \mathrm{Ar}-\mathrm{H})$, 8.06 (d, $J=8.4 \mathrm{~Hz}, 1 \mathrm{H}, \mathrm{Ar}-\mathrm{H}), 8.20$ (s, 1H, Ar-H), 8.39 (d, $J=8.4 \mathrm{~Hz}, 1 \mathrm{H}, \mathrm{Ar}-\mathrm{H}), 8.43(\mathrm{~s}, 1 \mathrm{H}$, pyridine $\mathrm{H} 4)$ and $11.22 \mathrm{ppm}(\mathrm{s}, 1 \mathrm{H}, \mathrm{NH}) ;{ }^{13} \mathrm{C}-\mathrm{NMR}$ (DMSO- $\left.d_{6}\right): \delta=14.49$ $\left(\mathrm{CH}_{3}\right), 24.38\left(\mathrm{CH}_{3}\right), 61.78\left(\mathrm{CH}_{2}\right), 113.36,119.16,126.98$, $128.44,129.16,131.98,132.11,132.78,133.57,135.32$, $135.57,141.21,147.48,148.45,158.16,165.63$ and 170.34 ppm (Ar-C and CO); MS (EI): m/z (\%) $501\left(\mathrm{M}^{+}, 100\right)$, $502\left(\mathrm{M}^{+}+1,72.45\right)$. Anal. calcd. for $\mathrm{C}_{22} \mathrm{H}_{17} \mathrm{Cl}_{2} \mathrm{~N}_{5} \mathrm{O}_{5}$ (502.32): C, 52.61; H, 3.41; N, 13.94. Found: C, 52.64; H, 3.37; N, 13.88 .

\section{2-Acetylamino-6-(4-chlorophenyl)-5-(4-chlorophenylazo) nicotinic acid ethyl ester (11b)}

Recrys- tallized from EtOH/dioxane (3:1) mixture as orange crystals, yield: (77\%), m.p. $103-104^{\circ} \mathrm{C}$; IR (KBr): $v / \mathrm{cm}^{-1} 3278(\mathrm{NH}), 1721,1685$ (CO and $\mathrm{CO}$ ester); ${ }^{1} \mathrm{H}-$ NMR (DMSO- $\left.d_{6}\right): \delta=1.30\left(\mathrm{t}, 3 \mathrm{H}, J=7.2 \mathrm{~Hz}, \mathrm{CH}_{3} \mathrm{CH}_{2}\right.$ ), $2.21\left(\mathrm{~s}, 3 \mathrm{H}, \mathrm{COCH}_{3}\right), 4.28$ (q, $2 \mathrm{H}, J=7.2 \mathrm{~Hz}, \mathrm{CH}_{3} \mathrm{CH}_{2}$ ), $7.63(\mathrm{~d}, J=8.4 \mathrm{~Hz}, 2 \mathrm{H}, \mathrm{Ar}-\mathrm{H}), 7.69$ (d, $J=8.4 \mathrm{~Hz}, 2 \mathrm{H}, \mathrm{Ar}-$ $\mathrm{H})$, 7.85-7.88 (m, $4 \mathrm{H}, \mathrm{Ar}-\mathrm{H}), 8.40(\mathrm{~s}, 1 \mathrm{H}$, pyridine $\mathrm{H} 4)$ and $11.10 \mathrm{ppm}$ (s, $1 \mathrm{H}, \mathrm{NH}) ; \mathrm{MS}(\mathrm{EI}): \mathrm{m} / \mathrm{z}(\%) 456\left(\mathrm{M}^{+}, 76.92\right)$, $457\left(\mathrm{M}^{+}+1\right.$, 67.22). Anal. calcd. for $\mathrm{C}_{22} \mathrm{H}_{18} \mathrm{Cl}_{2} \mathrm{~N}_{4} \mathrm{O}_{3}$ (457.32): C, 57.78; H, 3.97; N, 12.25. Found: C, 57.86; H, $3.88 ; \mathrm{N}, 12.27$.

\section{2-Acetylamino-6-(4-chlorophenyl)-5-(3-chlorophenylazo) nicotinic acid ethyl ester (11c)}

Recrystallized from EtOH/dioxane (3:1) mixture as orange crystals, yield: $(79 \%)$, m.p. $210-211^{\circ} \mathrm{C}$; IR (KBr): $v / \mathrm{cm}^{-1}$ $3245(\mathrm{NH}), 1709,1680(2 \mathrm{CO}) ;{ }^{1} \mathrm{H}-\mathrm{NMR}\left(\mathrm{DMSO}-d_{6}\right): \delta=$ $1.30\left(\mathrm{t}, 3 \mathrm{H}, J=7.2 \mathrm{~Hz}, \mathrm{CH}_{3} \mathrm{CH}_{2}\right), 2.21\left(\mathrm{~s}, 3 \mathrm{H}, \mathrm{COCH}_{3}\right)$, $4.28\left(\mathrm{q}, 2 \mathrm{H}, J=7.2 \mathrm{~Hz}, \mathrm{CH}_{3} \mathrm{CH}_{2}\right.$ ), 7.62-7.66 (m, 4H, Ar$\mathrm{H})$, 7.81-7.87 (m, 4H, Ar-H), 8.39 (s, 1H, pyridine $\mathrm{H} 4)$ and $11.11 \mathrm{ppm}(\mathrm{s}, 1 \mathrm{H}, \mathrm{NH}) ;{ }^{13} \mathrm{C}-\mathrm{NMR}\left(\mathrm{DMSO}-d_{6}\right): \delta=$ 14.04 $\left(\mathrm{CH}_{3}\right), 23.91\left(\mathrm{CH}_{3}\right), 61.27\left(\mathrm{CH}_{2}\right), 122.17,124.06$, $126.98,127.98,131.40,132.93,134.21,134.86,135.02$, $136.95,139.80,140.70,142.90,150.33,156.69,165.26$ and $169.75 \mathrm{ppm}$ (Ar-C and CO); MS (EI): $\mathrm{m} / \mathrm{z}$ (\%) $456\left(\mathrm{M}^{+}, 91.14\right), 457\left(\mathrm{M}^{+}+1,85.08\right)$. Anal. calcd. for $\mathrm{C}_{22} \mathrm{H}_{18} \mathrm{Cl}_{2} \mathrm{~N}_{4} \mathrm{O}_{3}$ (457.32): C, 57.78; $\mathrm{H}, 3.97 ; \mathrm{N}, 12.25$. Found: C, 57.69; H, 3.93; N, 12.31 .

\section{5-(2-Chloro-5-nitrophenylazo)-6-(4-chlorophenyl)-2- diacetylaminonicotinic acid ethyl ester (12)}

Recrystallized from EtOH as red crystals, yield: (89\%), m.p. $229-230^{\circ} \mathrm{C}$; IR (KBr): $v / \mathrm{cm}^{-1} 3231(\mathrm{NH}), 1720$, 1705, 1681 (3 CO); ${ }^{1} \mathrm{H}-\mathrm{NMR}$ (DMSO-d $): \delta=1.31$ $\left(\mathrm{t}, 3 \mathrm{H}, J=7.2 \mathrm{~Hz}, \mathrm{CH}_{3} \mathrm{CH}_{2}\right), 2.31\left(\mathrm{~s}, 6 \mathrm{H}, 2 \mathrm{COCH}_{3}\right)$, $4.36\left(\mathrm{q}, 2 \mathrm{H}, J=7.2 \mathrm{~Hz}, \mathrm{CH}_{3} \mathrm{CH}_{2}\right), 7.64(\mathrm{~d}, J=8.4$ $\mathrm{Hz}, 2 \mathrm{H}, \mathrm{Ar}-\mathrm{H}), 7.91$ (d, $J=8.4 \mathrm{~Hz}, 2 \mathrm{H}, \mathrm{Ar}-\mathrm{H}), 8.10$ (d, $J=8.4 \mathrm{~Hz}, 1 \mathrm{H}, \mathrm{Ar}-\mathrm{H}), 8.23(\mathrm{~s}, 1 \mathrm{H}, \mathrm{Ar}-\mathrm{H}), 8.45$ $(\mathrm{d}, J=8.4 \mathrm{~Hz}, 1 \mathrm{H}, \mathrm{Ar}-\mathrm{H})$ and $8.64 \mathrm{ppm}(\mathrm{s}, 1 \mathrm{H}$, pyridine $\mathrm{H} 4) ;{ }^{13} \mathrm{C}-\mathrm{NMR}$ (DMSO-d $): \delta=14.34\left(\mathrm{CH}_{3}\right), 26.75\left(\mathrm{CH}_{3}\right)$, $62.67\left(\mathrm{CH}_{2}\right), 113.56,124.98,127.68,128.44,128.73$, $129.79,132.93,133.57,133.76,134.51,136.14,141.32$, 144.73, 147.51, 148.43, 152.96, 158.34, 163.37 and 172.37 ppm (Ar-C and CO); MS (EI): m/z (\%) $543\left(\mathrm{M}^{+}, 8.55\right), 544$ $\left(\mathrm{M}^{+}+1,2.95\right)$. Anal. calcd. for $\mathrm{C}_{24} \mathrm{H}_{19} \mathrm{Cl}_{2} \mathrm{~N}_{5} \mathrm{O}_{6}$ (544.35): $\mathrm{C}$, 52.96; H, 3.52; N, 12.87. Found: C, 53.02; H, 3.45; N, 12.94 .

\section{General procedure for the preparation of amidines $13 a-b$}

Independent mixtures of the 2-amino-5-arylazonicotinates 8d,f (5 mmol), N,N-dimethylformamide dimethylacetal (DMF-DMA) $(0.6 \mathrm{~mL}, 5 \mathrm{mmol})$ in dry toluene $(20 \mathrm{~mL})$ were stirred at reflux for $4 \mathrm{~h}$. The separated solid product obtained on standing at room temperature was collected by filtration, washed by $\mathrm{EtOH}$ and recrystallized from dioxane to afford the corresponding amidines $\mathbf{1 3 a}, \mathbf{b}$ as pure products.

\section{5-(3-Bromophenylazo)-6-(4-chlorophenyl)-2-(dimethyl- aminomethyleneamino)nicotinic acid ethyl ester (13a)} reddish brown crystals, yield: (72\%), m.p. $226 .-227^{\circ} \mathrm{C}$; IR (KBr): $v / \mathrm{cm}^{-1} 1727$ (CO ester); ${ }^{1} \mathrm{H}-\mathrm{NMR}$ (DMSO- $\left.d_{6}\right): \delta=$ $1.33\left(\mathrm{t}, 3 \mathrm{H}, J=7.2 \mathrm{~Hz}, \mathrm{CH}_{3} \mathrm{CH}_{2}\right), 3.10\left(\mathrm{~s}, 3 \mathrm{H}, \mathrm{CH}_{3}\right), 3.21$ $\left(\mathrm{s}, 3 \mathrm{H}, \mathrm{CH}_{3}\right), 4.34$ (q, $\left.2 \mathrm{H}, J=7.2 \mathrm{~Hz}, \mathrm{CH}_{3} \mathrm{CH}_{2}\right), 7.56-770$ $(\mathrm{m}, 4 \mathrm{H}, \mathrm{Ar}-\mathrm{H}), 7.77-7.88(\mathrm{~m}, 4 \mathrm{H}, \mathrm{Ar}-\mathrm{H}), 8.21(\mathrm{~s}, 1 \mathrm{H}$, amidine $\mathrm{H}$ ) and $8.75 \mathrm{ppm}(\mathrm{s}, 1 \mathrm{H}$, pyridine $\mathrm{H} 4)$; $\mathrm{MS}$ (EI): $\mathrm{m} / \mathrm{z}$ (\%) 514 ( $\left.\mathrm{M}^{+}, 100\right), 515\left(\mathrm{M}^{+}+1,42.57\right)$. Anal. calcd. for $\mathrm{C}_{23} \mathrm{H}_{21} \mathrm{BrClN}_{5} \mathrm{O}_{2}$ (514.81): C, 53.66; H, 4.11; N, 13.60 . Found: C, 53.74; H, 4.15; N, 13.53. 
5-(2-Chloro-5-nitrophenylazo)-6-(4-chlorophenyl)-2(dimethylaminomethyleneamino)nicotinic acid ethyl ester (13b) red crystals, yield: (79\%), m.p. $196-197^{\circ} \mathrm{C}$; IR (KBr): $v / \mathrm{cm}^{-1}$ 1745 (CO ester); ${ }^{1} \mathrm{H}-\mathrm{NMR}$ (DMSO- $\left.d_{6}\right): \delta=1.33(\mathrm{t}, 3 \mathrm{H}, J=$ $7.2 \mathrm{~Hz}, \mathrm{CH}_{3} \mathrm{CH}_{2}$ ), 3.12 (s, $\left.3 \mathrm{H}, \mathrm{CH}_{3}\right), 3.23\left(\mathrm{~s}, 3 \mathrm{H}, \mathrm{CH}_{3}\right), 4.33$ $\left(\mathrm{q}, 2 \mathrm{H}, J=7.2 \mathrm{~Hz}, \mathrm{CH}_{3} \mathrm{CH}_{2}\right), 7.57$ (d, $\left.J=8.8 \mathrm{~Hz}, 2 \mathrm{H}, \mathrm{Ar}-\mathrm{H}\right)$, $7.91(\mathrm{~d}, J=8.8 \mathrm{~Hz}, 2 \mathrm{H}, \mathrm{Ar}-\mathrm{H}), 7.99(\mathrm{~d}, J=8.4 \mathrm{~Hz}, 1 \mathrm{H}$, Ar-H), 8.16 (s, $1 \mathrm{H}, \mathrm{Ar}-\mathrm{H}), 8.23$ (s, $1 \mathrm{H}$, amidine $\mathrm{H}), 8.30$ $(\mathrm{d}, J=8.4 \mathrm{~Hz}, 1 \mathrm{H}, \mathrm{Ar}-\mathrm{H})$ and $8.82 \mathrm{ppm}(\mathrm{s}, 1 \mathrm{H}$, pyridine $\mathrm{H} 4) ;$ ); ${ }^{13} \mathrm{C}-\mathrm{NMR}$ (DMSO- $\left.d_{6}\right): \delta=14.52\left(\mathrm{CH}_{3} \mathrm{CH}_{2}\right), 35.29$ $\left(\mathrm{CH}_{3}\right), 40.84\left(\mathrm{CH}_{3}\right), 61.23\left(\mathrm{CH}_{3} \mathrm{CH}_{2}\right), 113.22,122.14$, $125.54,125.92,128.08,132.50,133.30,134.95,136.80$, $139.98,140.08,147.72,149.14,157.25,158.55,162.17$ and $166.99 \mathrm{ppm}$ (Ar-C and CO); MS (EI): m/z (\%) 514 $\left(\mathrm{M}^{+}, 100\right), 515\left(\mathrm{M}^{+}+1,68.14\right)$. Anal. calcd. for $\mathrm{C}_{23} \mathrm{H}_{20} \mathrm{Cl}_{2} \mathrm{~N}_{6} \mathrm{O}_{4}$ (515.36): C, 53.60; H, 3.91; N, 16.31 . Found: C, 53.57; H, 3.87; N, 16.24 .

\section{General Procedure for the Preparation of pyrido[2,3-d] pyrimidin-4-one $15 a-b$}

Independent solutions of the amidines 13a,b $(5 \mathrm{mmol})$ in $\mathrm{AcOH}(20 \mathrm{~mL})$ containing ammonium acetate $(1.5 \mathrm{~g})$ were stirred at reflux for $4 \mathrm{~h}$. Then, the reaction mixtures were cooled to room temperature and poured onto ice cold water. The crude products were collected by filtration, washed with water and recrystallized from the appropriate solvent to afford the pyrido[2,3-d]pyrimidin derivatives 15a,b.

\section{6-(3-Bromophenylazo)-7-(4-chlorophenyl)-3H-pyrido[2,3-d] pyrimidin-4-one (15a)}

Reddish brown crystals, yield: (70\%), m.p. $290-291^{\circ} \mathrm{C}$; IR (KBr): $v / \mathrm{cm}^{-1} 3201(\mathrm{NH}), 1707$ (CO); ${ }^{1} \mathrm{H}-\mathrm{NMR}$ $\left(\mathrm{DMSO}-d_{6}\right): \delta=7.57-7.64(\mathrm{~m}, 3 \mathrm{H}, \mathrm{Ar}-\mathrm{H}), 7.79-7.88(\mathrm{~m}$, $4 \mathrm{H}, \mathrm{Ar}-\mathrm{H}), 7.95(\mathrm{~s}, 1 \mathrm{H}, \mathrm{Ar}-\mathrm{H}), 8.46(\mathrm{~s}, 1 \mathrm{H}$, pyrimidine $\mathrm{H} 2), 8.63(\mathrm{~s}, 1 \mathrm{H}$, pyridine $\mathrm{H} 5)$ and $12.78 \mathrm{ppm}(\mathrm{s}, 1 \mathrm{H}$, $\mathrm{NH}) ;{ }^{13} \mathrm{C}-\mathrm{NMR}$ (DMSO- $\left.d_{6}\right): \delta=117.54,122.58,122.74$, $123.05,125.18,127.98,131.67,133.01,134.59,134.99$, 135.56, 143.13, 150.50, 153.10, 159.76, 160.48 and 161.66 ppm (Ar-C and CO); MS (EI): m/z (\%) $440\left(\mathrm{M}^{+}, 100\right), 441$ $\left(\mathrm{M}^{+}+1\right.$, 36.87). Anal. calcd. for $\mathrm{C}_{19} \mathrm{H}_{11} \mathrm{BrClN}_{5} \mathrm{O}$ (440.69): C, 51.79; H, 2.52; N, 15.89. Found: C, 51.87; H, 2.46; N, 15.92 .

\section{6-(2-Chloro-5-nitrophenylazo)-7-(4-chlorophenyl)-3H-pyrido [2,3-d]pyrimidin-4-one (15b)}

Reddish brown crystals, yield: (74\%), m.p. above $300^{\circ} \mathrm{C}$; IR $(\mathrm{KBr}): v / \mathrm{cm}^{-1} 3212(\mathrm{NH}), 1702(\mathrm{CO}) ;{ }^{1} \mathrm{H}-\mathrm{NMR}$ $\left(\mathrm{DMSO}-d_{6}\right): \delta=7.62(\mathrm{~d}, J=8.4 \mathrm{~Hz}, 2 \mathrm{H}, \mathrm{Ar}-\mathrm{H}), 7.87(\mathrm{~d}$, $J=8.4 \mathrm{~Hz}, 2 \mathrm{H}, \mathrm{Ar}-\mathrm{H}), 8.04(\mathrm{~d}, J=8.8 \mathrm{~Hz}, 1 \mathrm{H}, \mathrm{Ar}-\mathrm{H})$, $8.16(\mathrm{~s}, 1 \mathrm{H}, \mathrm{Ar}-\mathrm{H}), 8.37$ (d, $J=8.8 \mathrm{~Hz}, 1 \mathrm{H}, \mathrm{Ar}-\mathrm{H}), 8.46$ $(\mathrm{s}, 1 \mathrm{H}$, pyrimidine $\mathrm{H} 2), 8.66(\mathrm{~s}, 1 \mathrm{H}$, pyridine $\mathrm{H} 5)$ and 12.74 $\mathrm{ppm}(\mathrm{s}, 1 \mathrm{H}, \mathrm{NH}) ;{ }^{13} \mathrm{C}-\mathrm{NMR}\left(\mathrm{DMSO}-d_{6}\right): \delta=$
$112.84,117.23,123.10,126.31,127.88,132.31,133.12$, $134.84,135.78,135.93,140.48,142.44,146.98,148.06$, 154.98, 158.82 and 160.94 ppm (Ar-C and CO); MS (EI): $\mathrm{m} / \mathrm{z}(\%) 440\left(\mathrm{M}^{+}, 100\right), 441\left(\mathrm{M}^{+}+1,55.15\right)$. Anal. calcd. for $\mathrm{C}_{19} \mathrm{H}_{10} \mathrm{Cl}_{2} \mathrm{~N}_{6} \mathrm{O}_{3}$ (441.24): C, 51.72; $\mathrm{H}, 2.28$; N, 19.05. Found: C, 51.68; H, 2.34; N, 19.13 .

\section{6-(2-Chloro-5-nitrophenylazo)-7-(4-chlorophenyl)2-thioxo- 2,3-dihydro-1H-pyrido[2,3-d]pyrimidin-4-one (16)}

A mixture of compound $\mathbf{8 f}(1.15 \mathrm{~g}, 2.5 \mathrm{mmol})$, thiourea $(0.2 \mathrm{~g}, 2.5 \mathrm{mmol})$, and a few drops from DMF was fused together in an oil bath under nitrogen gas at $250^{\circ} \mathrm{C}$ for 15 min, the fused mass was dissolved in DMF and poured onto ice cold water, the solid obtained was recrystallized from dioxane/DMF (2:1) to give compounds 16 as brown crystals, yield: (64\%), m.p. above $300^{\circ} \mathrm{C}$; IR (KBr): $v / \mathrm{cm}^{-1}$ 3326, $3264(2 \mathrm{NH}), 1701$ (CO); ${ }^{1} \mathrm{H}-\mathrm{NMR}$ $\left(\mathrm{DMSO}-d_{6}\right): \delta=7.57(\mathrm{~d}, J=8.4 \mathrm{~Hz}, 2 \mathrm{H}, \mathrm{Ar}-\mathrm{H}), 7.85(\mathrm{~d}, J=$ $8.4 \mathrm{~Hz}, 2 \mathrm{H}, \mathrm{Ar}-\mathrm{H}), 8.13$ (d, $J=8.4 \mathrm{~Hz}, 1 \mathrm{H}, \mathrm{Ar}-\mathrm{H}), 8.24$ (s, $1 \mathrm{H}, \mathrm{Ar}-\mathrm{H}), 8.41$ (d, $J=8.4 \mathrm{~Hz}, 1 \mathrm{H}, \mathrm{Ar}-\mathrm{H}), 8.59$ (s, $1 \mathrm{H}$, pyridine $\mathrm{H} 5), 9.17$ (s, $1 \mathrm{H}, \mathrm{NH})$, and $12.45 \mathrm{ppm}(\mathrm{s}, 1 \mathrm{H}$, $\mathrm{NH}) ; \mathrm{MS}(\mathrm{EI}): \mathrm{m} / \mathrm{z}$ (\%) $472\left(\mathrm{M}^{+}, 58.56\right), 473\left(\mathrm{M}^{+}+1\right.$, 16.77). Anal. calcd. for $\mathrm{C}_{19} \mathrm{H}_{10} \mathrm{Cl}_{2} \mathrm{~N}_{6} \mathrm{O}_{3} \mathrm{~S}$ (473.30): C, 48.22; H, 2.13 N, 17.76; S, 6.77. Found: C, 48.10; H, $2.17 ; \mathrm{N}, 17.65 ; \mathrm{S}, 6.62$.

\section{Antimicrobial evaluation \\ Methodology}

The antimicrobial activities of newly synthesized 22 different chemical compounds were tested using the Agar-well diffusion technique (Isaacson and Kirchbaum) [37] against five different microbial cultures. Pure cultures of E. coli (Culture \#0680P, Microbiologics, St. Cloud, MN, USA) and P. aeruginosa (Culture \#0416P, Microbiologics) (Gram-negative bacteria), B. subtilis (Culture \#0269P, Microbiologics), and S. aureus (Culture \#0831P, Microbiologics) (Gram-positive bacteria) and C. albicans (yeast) (Culture \#155965, Carolina Biological Supply, Burlington, NC, USA) were involved in the test. Bacterial strain cultures were cultivated in MuellerHinton broth (Difco) for all the bacterial strains after 24 $\mathrm{h}$ of incubation at $37^{\circ} \mathrm{C}$. The yeasts were propagated in Sabouraud dextrose broth (Difco) after incubation for 24 $\mathrm{h}$ at $25^{\circ} \mathrm{C},[38,39]$ an aliquot of $0.1 \mathrm{ml}$ of each bacterial strain was inoculated and spread on nutrient agar (NA), while $0.1 \mathrm{ml}$ of the yeast was spread on potato dextrose agar (PDA). The inoculated plates were supplied with $100 \mu \mathrm{l}$ of each of the tested chemicals with a total final concentration of $1 \mathrm{mg} \mathrm{ml}^{-1}$. The chemicals were included in 4-mm wells produced by a sterile cork borer. The NA plates were incubated at $37^{\circ} \mathrm{C}$ for $24 \mathrm{~h}$ while PDA plates were incubated at $25^{\circ} \mathrm{C}$ for $48 \mathrm{~h}$. The zones of inhibition around the wells were determined and the average based on three replicas was recorded. Ampicillin 
and cycloheximide (Sigma, St. Louis, MO, USA) both used as references in the experiment where ampicillin was used as an antibacterial drug, which is known to inhibit prokaryotes organisms while cycloheximide was used as an antifungal drug, which is known to inhibit eukaryotic organisms. The MIC measurement was determined for compounds with inhibition zones $>12 \mathrm{~mm}$ using a two-fold serial dilution technique [40]. The inhibition zone diameters values cited in Table 4 are attributed to the tested original concentration $(1 \mathrm{mg} / \mathrm{mL})$ as a preliminary test and the MIC $(\mu \mathrm{g} / \mathrm{mL})$ values are recorded in Table 5.

\section{MIC measurement}

The microdilution susceptibility test in Müllere-Hinton Broth (Difco) and Sabouraud dextrose broth (Difco) at $\mathrm{pH} 7.4$ was used for the determination of the antibacterial and antifungal activities, respectively $[38,39,41]$. Stock solutions of the tested compounds, ampicillin and cycloheximide, were prepared in DMSO at a concentration of $1000 \mu \mathrm{g} / \mathrm{mL}$. Each stock solution was diluted to prepare serial two-fold dilutions at concentrations in the range of $500-3.125 \mu \mathrm{g} / \mathrm{mL}$. The microorganism suspensions at approximately $10^{5} \mathrm{CFU} / \mathrm{mL}$ concentrations were inoculated to the corresponding 96-well plates. The sealed microplates were incubated at $37^{\circ} \mathrm{C}$ for $24 \mathrm{~h}$ for antibacterial activity and at $25^{\circ} \mathrm{C}$ for $48 \mathrm{~h}$ for antifungal activity in a humid chamber. At the end of the incubation period, the MIC values were recorded as the lowest concentrations of the substance that will inhibit the visible growth of the microorganisms (had no visible turbidity). Control experiments with DMSO and uninoculated media were run parallel to the tested compounds under the same conditions.

\section{Conclusions}

In this study, a general rule for the synthesis of 2-amino-5arylazo-6-aryl substituted nicotinic acids and pyridazinones was established using 3-oxo-2-arylhydrazonopropanals as precursors. Moreover, a novel route to pyrido[2,3- $d]$ pyrimidine was achieved. The antimicrobial activities of the resulting novel 2-amino-5-arylazonicotinates, pyridazinone, and pyrido[2,3- $-d]$ pyrimidine derivatives were investigated with the hope of discovering new structure leads that could serve as antibacterial and antifungal agents. The results of the antimicrobial activities revealed that most of these compounds were found to exhibit strong inhibitory effects on the growth of the Gram-positive bacteria especially B. subtilis. Compounds 1a, 8a-g, 10a-c, 15b, and $\mathbf{1 6}$ showed considerable antimicrobial activity against $B$. subtilis (Gram-positive bacteria). The results of biological evaluations demonstrated that most of these compounds had promising antimicrobial activities against Grampositive bacteria.

\section{Additional files}

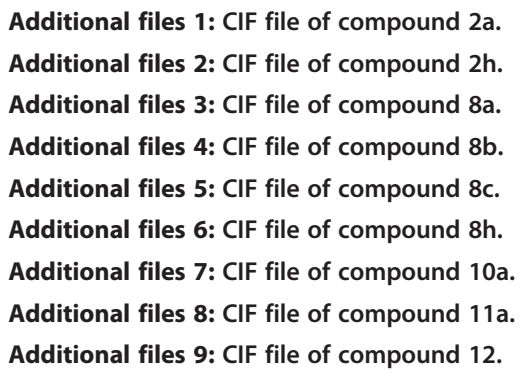

\section{Competing interests}

The authors declare that they have no competing interests.

\section{Authors' contributions}

The current study is an outcome of the constructive discussion between all authors. HMl carried out the synthesis, purification, and characterization of the compounds by MS, ${ }^{1} \mathrm{H}$ NMR, ${ }^{13} \mathrm{C}$ NMR spectral analyses, and the $X$-ray single crystal analysis. All the authors read and approved the final manuscript.

\section{Acknowledgments}

Support of this work was provided by the University of Kuwait through a research grant (SC03/11). The facilities of Analab/SAF supported by research grants GS01/01, GS01/05, GS01/03, and GS03/08 are gratefully acknowledged.

\section{Author details}

${ }^{1}$ Chemistry Department, Faculty of Science, Kuwait University, P.O. Box 5969, Safat 13060, Kuwait. ${ }^{2}$ Chemistry Department, Faculty of Science, Fayoum University, Fayoum 63514A. R., Egypt.

Received: 2 May 2013 Accepted: 4 July 2013

Published: 17 July 2013

\section{References}

1. Tolaro K, Tolaro A: Foundation of Microbiology. 3rd edition. Dubuque: W.C. Brown Publisher; 1993.

2. Gaonkar SL, Rai KML, Prabhuswamy B: Synthesis of novel 3-[5-ethyl-2-(2phenoxy-ethyl)-pyridin]-5-substituted isoxazoline libraries via 1,3-dipolar cycloaddition and evaluation of antimicrobial activities. Med Chem Res 2007, 15:407-417.

3. Mojahidul I, Anees AS, Ramadoss R: Synthesis, antitubercular, antifungal and antibacterial activities of 6-substituted phenyl-2-(3'-substituted phenyl pyridazin-6'-yl)-2,3,4,5-tetrahydropyridazin-3-one. Acta Pol Pharm 2008, 65:353-362

4. Kandile NG, Mohamed MI, Zaky H, Mohamed HM: Novel pyridazine derivatives: Synthesis and antimicrobial activity evaluation. Eur J Med Chem 2009, 44:1989-1996.

5. Butnariu RM, Caprosu MD, Bejan V, Mangalagiu II, Ungureanu M, Poiata A, Tuchilus C, Florescu M: Pyridazine and phthalazine derivatives with potential antimicrobial activity. J. Heterocyclic Chem 2007, 44:1149-1152.

6. Suksrichavalit T, Prachayasittikul S, Nantasenamat C, Isarankura-Na-Ayudhya C, Prachayasittikul V: Copper complexes of pyridine derivatives with superoxide scavenging and antimicrobial activities. Eur J Med Chem 2009, 44:3259-3265.

7. Hosni HM, Abdulla MM: Anti-inflammatory and analgesic activities of somenewly synthesized pyridinedicarbonitrile and benzopyranopyridine derivatives. Acta Pharm 2008, 58:175-186.

8. Dogruer DS, Unlu S, Kupeli E, Banoglu E, Sahin MF: Synthesis of 2-[5,6diphenyl-3(2H)-pyridazinone-2-yl]acetamide and 3-[5,6-diphenyl-3(2H)pyridazinone-2-yl]propanamide derivatives as analgesic and anti inflamematory agents. Turkish J Pharm Sci 2007, 4:57-70.

9. Gökçe M, Colak MS, Küpeli E, Sahin MF: Synthesis and analgesic and antiinflammatory activity of 6-phenyl/(4-methylphenyl)-3(2H)-pyridazinon-2propionamide derivatives. Arzneimittelforschung 2009, 59:357-363. 
10. Ali MA, Yar MS, Siddiqui AA, Sriram D, Yogeeswari P, De Clercq E: Synthesis and anti-HIV activity of N'-nicotinoyl-3-(4'-hydroxy-3'-methylphenyl)-5[substituted phenyl]-2-pyrazo- lines. Acta Pol Pharm 2007, 64:423-428.

11. Kumar S, Das SK, Dey S, Maity P, Guha M, Choubey V, Panda G, Bandyopadhyay U: Antiplasmodial activity of [(aryl)arylsulfanylmethyl] pyridine. Antimicrob Agents Chemother 2008, 52:705-715.

12. Lourenço MCS, de Souza MVN, Pinheiro AC, de ML F, Goncalves RSB, Nogueira TCM, Peralta MA: Evaluation of anti-tubercular activity of nicotinic and isoniazid analogues. ARKIVOC 2007, xv:181-191.

13. Sharma PC, Jain S: Synthesis and in vitro antibacterial activity of some novel N-nicotinoyl-1-ethyl-6-fluoro-1,4-dihydro-7-piperazin-1-yl-4oxoquinoline-3-carboxylates. Acta Pol Pharm 2008, 65:551-556.

14. Shafiee A, Rastkari N, Sharifzadeh M: Anticonvulsant activities of new 1,4-dihydropyridine derivatives containing 4-nitroimidazolyl substituents. Daru 2004, 12:81-86.

15. Rubat $C$, Coudert $P$, Refouvelet $B$, Tronche $P$, Bastide $P$, Bastide J: Anticonvulsant activity of 3-oxo-5-substituted benzylidene-6-methyl(4H)-2-pyridazinylacetamides and 2-pyridazinyl- acetylhydrazides. Chem Pharm Bull 1990, 38:3009-3013.

16. Chintakunta VK, Akella V, Vedula MS, Mamnoor PK, Mishra P, Casturi SR, Vangoori A, Rajagopalan R: 3-O-Substituted benzyl pyridazinone derivatives as COX inhibitors. Eur J Med Chem 2002, 37:339-347.

17. Rathish IG, Javed K, Bano S, Ahmad S, Alam MS, Pillai KK: Synthesis and blood glucose lowering effect of novel pyridazinone substituted benzenesulfonylurea derivatives. Eur J Med Chem 2009, 44:2673-2678.

18. Barbaro R, Betti L, Botta M, Corelli F, Giannaccini G, Maccari L, Manetti F, Strappaghetti G, Corsano S: Synthesis, biological evaluation, and pharmacophore generation of new pyridazinone derivatives with affinity toward $a_{1}$ - and $a_{2}$-adrenoceptors ${ }^{1}$. J Med Chem 2001, 44:2118-2132.

19. Miguel FB, Monica CMLG, Elena PM, Berta L, de PT B, Ana R, Nuria A Francisco L, Dolores MM, Olivier L, Laurent M: Pyrazolo [3,4-c]pyridazines as novel and selective inhibitors of cyclin-dependent kinases. J Med Chem 2005, 48:6843-6854.

20. Malinka W, Redzicka A, Lozach O: New derivatives of pyrrolo $[3,4-d]$ pyridazinone and their anticancer effects. Farmaco 2004, 59:457-462

21. Thapa P, Karki R, Thapa U, Jahng Y, Jung M-J, Nam JM, Na Y, Kwon Y, Lee ES: 2-Thienyl-4-furyl-6-aryl pyridine derivatives: Synthesis, topoisomerase I and II inhibitory activity, cytotoxicity, and structure-activity relationship study. Bioorg Med Chem 2010, 18:377-386.

22. Thapa P, Karki R, Choi H, Choi JH, Yun M, Jeong B-S, Jung M-J, Nam JM, Na Y, Cho W-J, Kwon Y, Lee E-S: Synthesis of 2-(thienyl-2-yl or -3-yl)-4-furyl6-aryl pyridine derivatives and evaluation of their topoisomerase I and II inhibitory activity, cytotoxicity, and structure activity relationship. Bioorg Med Chem 2010, 18:2245-2254.

23. Sotelo E, Fraiz N, Yanez M, Terrades V, Laguna R, Cano E, Ravina E: Pyridazines. Part XXIX: synthesis and platelet aggregation inhibition activity of 5-substituted-6-phenyl-3(2H)-pyridazinones novel aspects of their biological action. Bioorg Med Chem 2002, 10:2873-2882

24. Griebel G, Perrault G, Sanger DJ: Differences in anxiolytic-like profile of two novel nonbenzodiazepine BZ (omega) receptor agonists on defensive behaviors of mice. Pharmacol Biochem Behav 1999, 62:689-694.

25. Wermuth CG, Schlewer G, Bourguignon JJ, Maghioros G, Bouchet MJ, Moire C, Kan JP, Worms P, Biziere K: 3-Aminopyridazine derivatives with atypical antidepressant, serotonergic, and dopaminergic activities. J Med Chem 1989, 32:528-537.

26. Caliskan EB, Sukuroglu M, Coban T, Banoglu E, Suzen S: Screening and evaluation of antioxidant activity of some pyridazine derivatives. J. Enz. Inhib. Med. Chem 2008, 23:225-229.

27. Wu J, Kang S, Luo L, Shi Q, Ma J, Yin J, Song B, Hu D, Yang S: Synthesis and antifungal activities of novel nicotinamide derivatives containing 1,3,4-oxadiazole. Chem Cent J 2013, 7:64.

28. Behbehani $\mathrm{H}$, Ibrahim HM, Makhseed S, Mahmoud H: Applications of 2-arylhydrazono- nitriles in synthesis: Preparation of new indole containing 1,2,3-triazole, pyrazole and pyrazolo[1,5-a]pyrimidine derivatives and evaluation of their antimicrobial activities. Eur J Med Chem 2011, 46:1813-1820.

29. Behbehani $\mathrm{H}$, Ibrahim HM, Makhseed S, Elnagdi MH, Mahmoud H: 2-Aminothiophenes as building blocks in heterocyclic synthesis: Synthesis and antimicrobial evaluation of a new class of pyrido[1,2-a] thieno[3,2-e]pyrimidine, quinoline and pyridin-2-one derivatives. Eur $]$ Med Chem 2012, 52:61-65.
30. Abdel-Khalik MM, Agamy SM, Elnagdi MH: Chemistry of 2-arylhydrazonopropanals: novel synthesis of 1,6-dihydropyridazines and 5-heteroaryl substituted pyrazolo[1,5-a]pyrimidines and pyrazolo[3,4- $b$ ]pyridines. Synthesis 2001, 12:1861-1865

31. Al-Mousawi SM, Moustafa MS, Abdelshafy IA, Elnagdi MH: Reassignment of the structures of condensation products of a-keto $a^{\prime}$ formylarylhydrazones with ethyl cyanoacetate: A novel route to ethyl 5-arylazo-2-hydroxynicotinates. Tetrahedron Lett 2011, 52(2):202-204.

32. Al-Mousawi SM, El-Apasery MA: Condensation reactions of 3-oxo-2arylhydrazono- propanals with active methylene reagents: formation of 2-hydroxy- and 2-amino-6-substituted-5-arylazonicotinates and pyrido $[3,2-c]$ cinnolines via $6 \pi$-electrocyclization reactions. Molecules 2012 , 17:6547-6556.

33. Sharma P, Rane N, Gurram VK: Synthesis and QSAR studies of pyrimido [4,5- $d$ ]pyrimidine-2,5-dione derivatives as potential antimicrobial agents. Bioorg Med Chem Lett 2004, 14:4185-4190.

34. CrystalStructure 4.0: Crystal Structure Analysis Package. Tokyo 196-8666, Japan: Rigaku Corporation; 2000-2010

35. Sheldrick GM: SHELX97. Acta Cryst 2008, A64:112-122.

36. The crystallographic data for $2 a$ (CCDC 913918), 2g (CCDC 902315), 8 a (CCDC 902672), 86 (CCDC 902317), 8c (CCDC 902316), 8h (CCDC 902673), 10a (CCDC 902318), 11 a (CCDC 903834) and 12 (CCDC 902671) can be obtained on request from the director. 12 Union Road, Cambridge CB2 1EW, UK: Cambridge Crystallographic Data Center (Table 6).

37. Isaacson DM, Kirschbaum J: Assays of antimicrobial substances. In Manual of Industrial Microbiology and Biotechnology. Edited by Demain AL, Solomon NA. Washington, DC, USA: ASM Press; 1986:410-435.

38. Oren I, Yalcın I, Sener E, Ucarturk N: Synthesis and structure-activity relationships of new antimicrobial active multisubstituted benzazole derivatives. Eur J Med Chem 2004, 39:291-298.

39. Arpaci OT, Oren I, Altanlar N: Synthesis and antimicrobial activity of some novel 2-(p-substituted-phenyl)-5-substitutedcarbonylaminobenzoxazoles. // Farmaco 2002, 57:175-181.

40. National Committee for Clinical Laboratory Standards Methods for Dilution Antimicrobial Susceptibility Tests for bacteria that grow aerobically: Approved Standard M7-A5. Villanova, Pa: NCCLS; 2000

41. Shamroukh AH, Zaki MEA, Morsy EMH, Abdel-Motti FM, Abdel-Megeid FME: Synthesis, isomerization, and antimicrobial evaluation of some pyrazolopyranotriazolopyrimidine derivatives. Arch Pharm Chem Life Sci 2007, 340:345-351.

\section{doi:10.1186/1752-153X-7-123}

Cite this article as: Ibrahim et al:: Approaches towards the synthesis of a novel class of 2-amino-5-arylazonicotinate, pyridazinone and pyrido[2,3d] pyrimidine derivatives as potent antimicrobial agents. Chemistry Central Journal 2013 7:123.

Publish with ChemistryCentral and every
scientist can read your work free of charge
"Open access provides opportunities to our
colleagues in other parts of the globe, by allowing
anyone to view the content free of charge."
W. Jeffery Hurst, The Hershey Company.
- available free of charge to the entire scientific community
- peer reviewed and published immediately upon acceptance
- cited in PubMed and archived on PubMed Central
- yours - you keep the copyright
submit your manuscript here:
http://www.chemistrycentral.com/manuscript/

